\title{
Breathing Pulses in an Excitatory Neural Network*
}

\author{
Stefanos E. Folias ${ }^{\dagger}$ and Paul C. Bressloff
}

\begin{abstract}
In this paper we show how a local inhomogeneous input can stabilize a stationary-pulse solution in an excitatory neural network. A subsequent reduction of the input amplitude can then induce a Hopf instability of the stationary solution resulting in the formation of a breather. The breather can itself undergo a secondary instability leading to the periodic emission of traveling waves. In one dimension such waves consist of pairs of counterpropagating pulses, whereas in two dimensions the waves are circular target patterns.
\end{abstract}

Key words. traveling waves, excitatory neural network, breathers, integro-differential equations

AMS subject classification. $92 \mathrm{C} 20$

DOI. $10.1137 / 030602629$

1. Introduction. Various in vitro experimental studies have observed waves of excitation propagating in cortical slices when stimulated appropriately $[7,12,35]$. The propagation velocity of these synaptically generated waves is of the order $0.06 \mathrm{~m} / \mathrm{s}$, which is much slower than the typical speed of $0.5 \mathrm{~m} / \mathrm{s}$ found for action-potential propagation along axons. Traveling waves of electrical activity have also been observed in vivo in the somatosensory cortex of behaving rats [24], turtle and mollusk olfactory bulbs [18, 21], turtle cortex [27], and visuomotor cortices in the cat [29]. Often these traveling waves occur during periods without sensory stimulation, with the subsequent presentation of a stimulus inducing a switch to synchronous oscillatory behavior [11]. This suggests that determining the conditions under which cortical wave propagation can occur is important for understanding the normal processing of sensory stimuli as well as more pathological forms of behavior such as epileptic seizures and migraines.

A number of theoretical studies have established the occurrence of traveling fronts $[8,15]$ and traveling pulses $[36,1,19,25,30]$ in one-dimensional excitatory neural networks modeled in terms of nonlinear integro-differential equations. Such equations are infinite dimensional dynamical systems and can be written in the general form [10]

$$
\begin{aligned}
\frac{\partial u(x, t)}{\partial t} & =-u(x, t)+\int_{-\infty}^{\infty} w\left(x \mid x^{\prime}\right) f\left(u\left(x^{\prime}, t\right)\right) d x^{\prime}-\beta q(x, t)+I(x), \\
\frac{1}{\epsilon} \frac{\partial q(x, t)}{\partial t} & =-q(x, t)+u(x, t),
\end{aligned}
$$

where $u(x, t)$ is a neural field that represents the local activity of a population of excitatory neurons at position $x \in \mathbf{R}, I(x)$ is an external input current, $f(u)$ denotes the output firing

\footnotetext{
*Received by the editors December 19, 2003; accepted for publication (in revised form) by D. Terman April 14, 2004; published electronically September 29, 2004.

http://www.siam.org/journals/siads/3-3/60262.html

${ }^{\dagger}$ Department of Mathematics, University of Utah, 155 S 1400 E, Salt Lake City, UT 84112 (sfolias@math.utah.edu, bressloff@math.utah.edu).
} 
rate function, and $w\left(x \mid x^{\prime}\right)$ is the strength of connections from neurons at $x^{\prime}$ to neurons at $x$. The neural field $q(x, t)$ represents some form of negative feedback mechanism such as spike frequency adaptation or synaptic depression, with $\beta, \varepsilon$ determining the relative strength and rate of feedback. The nonlinear function $f$ is typically taken to be a sigmoid function $f(u)=$ $1 /\left(1+\mathrm{e}^{-\gamma(u-\kappa)}\right)$ with gain $\gamma$ and threshold $\kappa$. It can be shown [25] that there is a direct link between the above model and experimental studies of wave propagation in cortical slices where synaptic inhibition is pharmacologically blocked $[7,12,35]$. Since there is strong vertical coupling between cortical layers, it is possible to treat a thin cortical slice as an effective onedimensional medium. Analysis of the model provides valuable information regarding how the speed of a traveling wave, which is relatively straightforward to measure experimentally, depends on various features of the underlying cortical circuitry.

One of the common assumptions in the analysis of traveling wave solutions of (1.1) is that the system is spatially homogeneous; that is, the external input $I(x)$ is independent of $x$ and the synaptic weights depend only on the distance between presynaptic and postsynaptic cells, $w\left(x \mid x^{\prime}\right)=w\left(x-x^{\prime}\right)$. The existence of traveling waves can then be established for a class of weight distributions $w(x)$ that includes the exponential function $\mathrm{e}^{-|x| / d}$. The waves are in the form of traveling fronts in the absence of any feedback [8], whereas traveling pulses tend to occur when there is significant feedback [25]. The real cortex, however, is more realistically modeled as an inhomogeneous medium. Inhomogeneities in the synaptic weight distribution $w$ may arise due to the patchy nature of long-range horizontal connections in superficial layers of cortex. For example, in the primary visual cortex the horizontal connections tend to link cells with similar stimulus feature preferences such as orientation and ocular dominance $[23,37,3]$. The variation of the feature preferences across the cortex is approximately periodic, and this induces a corresponding periodic modulation in the horizontal connections. It has previously been shown that an inhomogeneous periodic modulation in the strength of synaptic interactions induced by long-range patchy connections can lead to wavefront propagation failure [4]. If the wavelength of the periodic inhomogeneity is much shorter than the characteristic wavelength of the front, then averaging theory can be used to achieve an effective homogenization of the neural medium along similar lines to that previously developed for a model of calcium waves [16] and for a model of chemical waves in a bistable medium [17].

Another important source of spatial inhomogeneity is the external input $I(x)$. Such inputs would arise naturally from sensory stimuli in the case of the intact cortex and could be introduced by external stimulation in the case of cortical slices. We have recently shown how a monotonically varying input can induce wave propagation failure due to the pinning of a stationary-front solution [5,6]. More significantly, the stationary front can subsequently destabilize via a Hopf bifurcation as the degree of input inhomogeneity is reduced, resulting in an oscillatory back-and-forth pattern of wave propagation. Analogous breather-like front solutions have previously been found in inhomogeneous reaction-diffusion systems $[28,31,13$, $14,2,26]$ and in numerical simulations of a realistic model of fertilization calcium waves [22].

In this paper we extend our work on fronts by analyzing the effects of input inhomogeneities on the stability of stationary pulses, since these better reflect the types of neural activity patterns observed in the cortex. In order to construct exact wave solutions, we follow previous treatments [1,25] and consider the high gain limit $\gamma \rightarrow \infty$ of the sigmoid function $f$ such that $f(u)=H(u-\kappa)$, where $H$ is the Heaviside step function; that is, $H(u)=1$ if $u \geq 0$ and 
$H(u)=0$ if $u<0$. As a further simplification, we also assume that the weight distribution $w$ is homogeneous so that (1.1) reduces to the form

$$
\begin{aligned}
\frac{\partial u(x, t)}{\partial t} & =-u(x, t)+\int_{-\infty}^{\infty} w\left(x-x^{\prime}\right) H\left(u\left(x^{\prime}, t\right)-\kappa\right) d x^{\prime}-\beta q(x, t)+I(x), \\
\frac{1}{\epsilon} \frac{\partial q(x, t)}{\partial t} & =-q(x, t)+u(x, t) .
\end{aligned}
$$

We first construct explicit traveling wave solutions of (1.2) in the case of a constant input (section 2). We then analyze the existence and stability of stationary pulses in the presence of a unimodal input (section 3). We show that (i) a sufficiently large input inhomogeneity can stabilize a stationary pulse and (ii) a subsequent reduction in the level of inhomogeneity can induce a Hopf instability of the stationary pulse leading to the formation of a breatherlike oscillatory wave. Numerically we find that a secondary instability can occur beyond which the breather periodically emits pairs of traveling pulses (section 4). Moreover, there is mode-locking between the oscillation frequency of the breather and the rate of wave emission. Analogous forms of oscillatory wave are also shown to occur in a more biophysically realistic conductance-based model (section 4). Finally, we extend our analysis to radially symmetric pulses in a two-dimensional network (section 5).

2. Traveling pulses in a homogeneous network. We begin by briefly outlining the construction of traveling pulse solutions of (1.2) in the case of zero input $I(x)=0$, following the approach of Pinto and Ermentrout [25]. We assume that the weight distribution $w(x)$ is a positive even function of $x$, is a monotonically decreasing function of $|x|$, and satisfies the normalization condition $\int_{-\infty}^{\infty} w(x) d x<\infty$. For concreteness, $w$ is taken to be an exponential weight distribution

$$
w(x)=\frac{1}{2 d} \mathrm{e}^{-|x| / d} .
$$

The length scale is fixed by setting $d=1$. Translation symmetry implies that we can consider traveling pulse solutions of the form $u(x, t)=U(x-c t)$ with $U( \pm \infty)=0$ and $U(-a)=$ $U(0)=\kappa$. Without loss of generality, we take $c>0$. Substituting into (1.2) with $I(x)=0$ and differentiating with respect to $\xi$ lead to the second-order boundary value problem

$$
\begin{aligned}
-c^{2} U^{\prime \prime}(\xi)+c[1+\epsilon] U^{\prime}(\xi)-\epsilon[1+\beta] U(\xi) & =c[w(\xi+a)-w(\xi)]+\epsilon[W(\xi+a)-W(\xi)], \\
U(0)=U(-a) & =\kappa \\
U( \pm \infty) & =0
\end{aligned}
$$

where $W(\xi)=\int_{0}^{\xi} w(x) d x$. Equation (2.2) is solved by considering separately the domains $\xi \leq-a,-a \leq \xi \leq 0$, and $\xi \geq 0$ and matching the solutions at $\xi=-a, 0$. On the domain $\xi>0$, with $w$ given by the exponential function (2.1),

$$
\begin{aligned}
-c^{2} U^{\prime \prime}(\xi)+c(1+\epsilon) U^{\prime}(\xi)-\epsilon(1+\beta) U(\xi) & =\frac{c+\epsilon}{2}\left(\mathrm{e}^{-\xi}-\mathrm{e}^{-(\xi+a)}\right), \\
U(0) & =\kappa, \\
U(\infty) & =0 .
\end{aligned}
$$


This has the solution $U_{>}(\xi)=\kappa \mathrm{e}^{-\xi}$ provided that

$$
\kappa=\frac{(c+\epsilon)\left(1-\mathrm{e}^{-a}\right)}{2\left(c^{2}+c(1+\epsilon)+\epsilon(1+\beta)\right)} \equiv f(c, a) .
$$

On the other two domains we have solutions consisting of complementary and particular parts:

$$
U_{0}(\xi)=\mathcal{A}_{+} \mathrm{e}^{\mu_{+} \xi}+\mathcal{A}_{-} \mathrm{e}^{\mu_{-} \xi}+\mathcal{U}_{+} \mathrm{e}^{\xi}+\mathcal{U}_{-} \mathrm{e}^{-\xi}
$$

for $-a<\xi<0$ and

$$
U_{<}(\xi)=\mathcal{A}_{+}^{\prime} \mathrm{e}^{\mu_{+} \xi}+\mathcal{A}_{-}^{\prime} \mathrm{e}^{\mu_{-} \xi}+\mathcal{U}_{+}^{\prime} \mathrm{e}^{\xi}
$$

for $\xi<-a$, where

$$
\mu_{ \pm}=\frac{1}{2 c}\left[1+\epsilon \pm \sqrt{(1+\epsilon)^{2}-4 \epsilon(1+\beta)}\right]
$$

The coefficients $\mathcal{U}_{ \pm}$and $\mathcal{U}_{+}^{\prime}$ are obtained by direct substitution into the differential equation for $U$, whereas the four coefficients $\mathcal{A}_{ \pm}$and $\mathcal{A}_{ \pm}^{\prime}$ are determined by matching solutions at the boundaries. This leads to the five boundary conditions (i) $U_{0}(0)=\kappa$, (ii) $U_{0}(-a)=\kappa$, (iii) $U_{<}(-a)=\kappa$, (iv) $U_{0}^{\prime}(0)=-\kappa$, and (v) $U_{0}^{\prime}(-a)=U_{<}^{\prime}(-a)$. Since there are five equations in four unknowns, we generate a second constraint on the speed and size of the wave that is of the form $g(c, a)=\kappa$. A full solution to the wave equation can then be found for just those values of $c, a$ which satisfy both $f(c, a)=\kappa$ and $g(c, a)=\kappa$.

Pinto and Ermentrout [25] used a shooting method to show that for sufficiently slow negative feedback (small $\epsilon$ ) and large $\beta$ there exist two pulse solutions, one narrow and slow and the other wide and fast. Numerically, the fast solution is found to be stable [25]. It is also possible to construct an explicit stationary-pulse solution by setting $c=0$ in (2.2):

$$
U(\xi)=\left\{\begin{array}{cc}
\frac{\mathrm{e}^{-\xi}}{2(1+\beta)}\left(1-\mathrm{e}^{-a}\right) & \text { for } \xi>0, \\
\frac{1}{2(1+\beta)}\left[2-\mathrm{e}^{\xi}-\mathrm{e}^{-(\xi+a)}\right] & \text { for }-a<\xi<0, \\
\frac{\mathrm{e}^{\xi}}{2(1+\beta)}\left(\mathrm{e}^{a}-1\right) & \text { for } \xi<-a
\end{array}\right.
$$

with

$$
\kappa=\frac{1}{2(1+\beta)}\left(1-\mathrm{e}^{-a}\right)
$$

It turns out that stationary-pulse solutions are unstable in the case of homogeneous inputs (see section 3), acting as separatrices between a zero activity state and a traveling wavefront. 
3. Stationary pulses in an inhomogeneous network. We now investigate the existence and stability of one-dimensional stationary pulses in the presence of a unimodal input $I(x)$ which, for concreteness, is taken to be a Gaussian of width $\sigma$ centered at the origin

$$
I(x)=\mathcal{I} \mathrm{e}^{-x^{2} / 2 \sigma^{2}} .
$$

As in section 2, we take $w$ to be a positive even function of $x$ and a monotonically decreasing function of $|x|$, and we choose the normalization $\int_{-\infty}^{\infty} w(x) d x=1$. For illustrative purposes, the exponential weight distribution (2.1) will be used as a specific example.

3.1. Stationary-pulse existence. From symmetry arguments there exists a stationarypulse solution $U(x)$ of (1.2) centered at $x=0$, satisfying

$$
\begin{aligned}
& U(x)>\kappa, \quad x \in(-a, a) \text {; } \\
& U( \pm a)=\kappa, \\
& U(x)<\kappa, \quad x \in(-\infty,-a) \cup(a, \infty) ; \\
& U( \pm \infty)=0 \text {. }
\end{aligned}
$$

In particular,

$$
(1+\beta) U(x)=\int_{-a}^{a} w\left(x-x^{\prime}\right) d x^{\prime}+I(x) .
$$

The threshold $\kappa$ and width $a$ are related according to the self-consistency condition

$$
\hat{\kappa}=[I(a)+W(2 a)] \equiv G(a),
$$

where $\hat{\kappa}=(1+\beta) \kappa$ and $W(2 a)=\int_{0}^{2 a} w(x) d x$. The existence or otherwise of a stationary-pulse solution can then be established by finding solutions to (3.3). Consider, for example, the exponential weight distribution (2.1) with $d=1$ such that $W(2 a)=\left(1-\mathrm{e}^{-2 a}\right) / 2$. Furthermore, suppose that the amplitude $\mathcal{I}$ of the Gaussian input (3.1) is treated as a bifurcation parameter with the range $\sigma$ kept fixed. (The effect of varying $\sigma$ will be discussed below.) It is straightforward to show that there always exists a critical amplitude $\mathcal{I}_{c}$, below which $G(a)$ is strictly monotonically increasing and above which $G(a)$ has two stationary points. Consequently, as $\hat{\kappa}$ varies, we have the possibility of zero, one, two, or three stationary-pulse solutions. The function $G(a)$ is plotted in Figure 1 for a range of input amplitudes $\mathcal{I}$, with horizontal lines indicating different values of $\hat{\kappa}$ : intersection points determine the existence of stationary-pulse solutions. Let $\kappa_{c}$ denote the value of $G(a)$ for which $G^{\prime}(a)$ has a double zero. Anticipating the stability results of section 3.2, we obtain the following results. If $\hat{\kappa}<\kappa_{c}$, then there is only a single pulse solution branch which is always unstable. On the other hand, if $\hat{\kappa}>\kappa_{c}$, then there are two distinct bifurcation scenarios (see Figure 2), both of which can support a stable pulse solution.

Scenario (i): $\kappa_{c}<\hat{\kappa}<1 / 2$. There exist three solution branches with the lower (narrow pulse) and upper (wide pulse) branches unstable. If $\epsilon>\beta$, then the middle (intermediate pulse) branch is stable along its entire length, annihilating in a saddle-node bifurcation at the endpoints $S, S^{\prime}$. On the other hand, if $\epsilon<\beta$, then only a central portion of the middle branch is stable due to the existence of two Hopf bifurcation points $H, H^{\prime}$. In the limit $\epsilon \rightarrow \beta$ we have $H \rightarrow S$ and $H^{\prime} \rightarrow S^{\prime}$ leading to some form of degenerate bifurcation. Note that as $\widehat{\kappa} \rightarrow 1 / 2$, $a_{S^{\prime}} \rightarrow \infty$, thus causing the upper branch to collapse. 


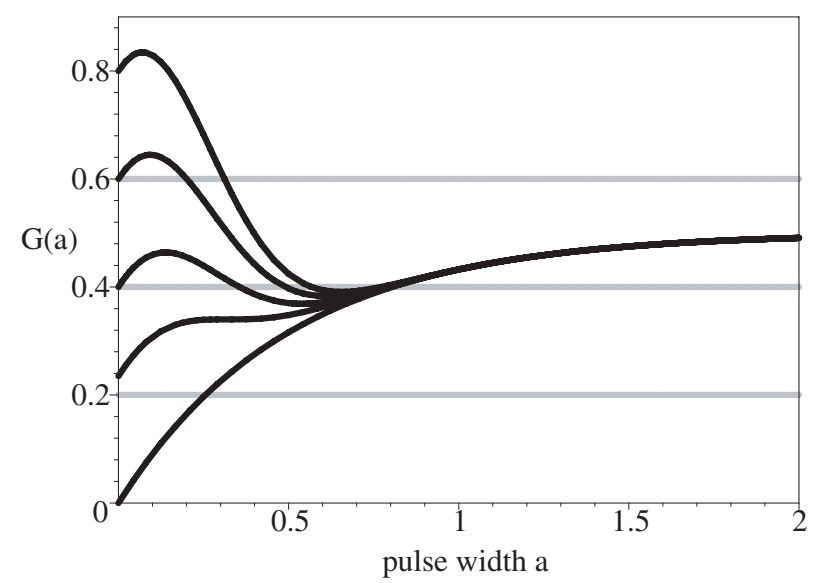

Figure 1. Plot of $G(a)$ in (3.3) as a function of pulse width a for an exponential weight distribution and various values of input amplitude $\mathcal{I}$ with $\sigma=0.25$. Horizontal lines (gray) represent different values of $\hat{\kappa}=\kappa(1+\beta)$. Intersections of black and gray curves indicate the existence of stationary-pulse solutions.
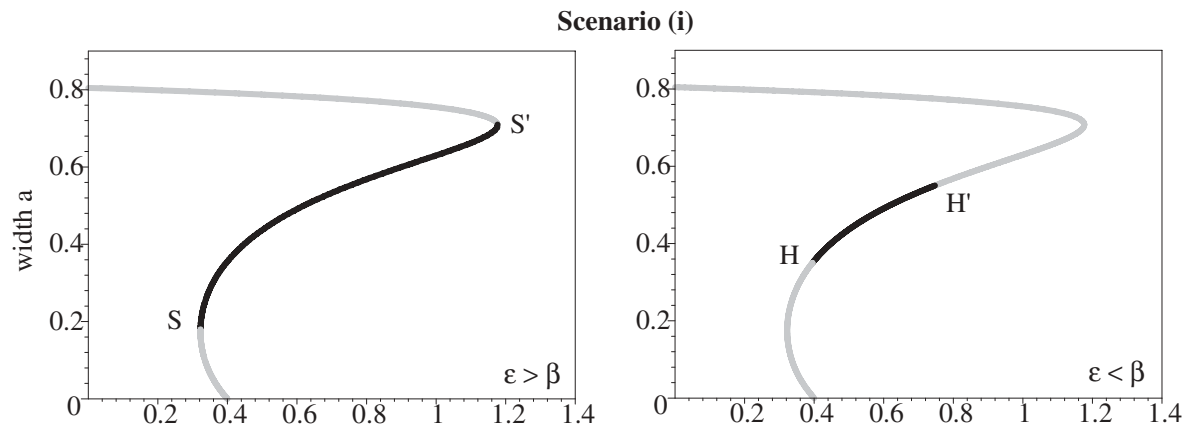

Scenario (ii)
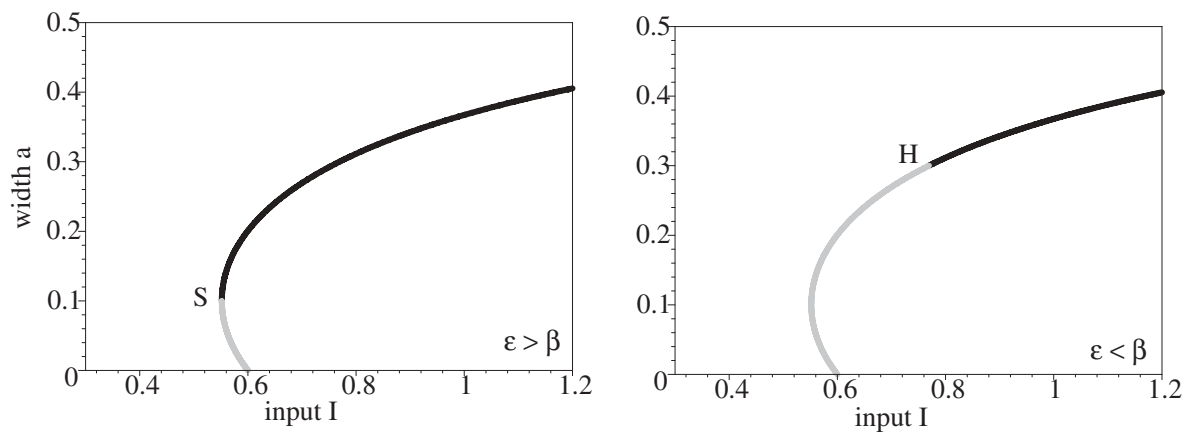

Figure 2. One-dimensional stationary-pulse existence curves for an exponential weight distribution and (i) $\kappa_{c}<\hat{\kappa}<\frac{1}{2}$, (ii) $\hat{\kappa}>\frac{1}{2}$. Other parameter values are $\beta=1, \sigma=0.25$. Black indicates stability, whereas gray indicates instability of the stationary pulse. Saddle-node bifurcation points are indicated by $S, S^{\prime}$ and $H o p f$ bifurcation points by $H, H^{\prime}$. 
Scenario (ii): $\hat{\kappa}>\mathbf{1 / 2}$. There exist two solution branches with the lower branch unstable and the upper branch stable for sufficiently large $\mathcal{I}$. If $\epsilon>\beta$, then the upper branch is stable along its entire length, annihilating in a saddle-node bifurcation at its endpoint $S$. On the other hand, if $\epsilon<\beta$, then the upper branch loses stability via a Hopf bifurcation at the point $H$ with $H \rightarrow S$ as $\epsilon \rightarrow \beta$.

In both of the above scenarios there also exists a stable subthreshold solution $U(x)=$ $I(x) /(1+\beta)$ when $\mathcal{I}<\hat{\kappa}$. This is coexistent with the lower suprathreshold pulse, and the pair annihilate at $\mathcal{I}=\hat{\kappa}$. To address the effect of varying the input $\sigma$, consider the case where $\hat{\kappa}<\frac{1}{2}$. As $\sigma$ decreases, $\kappa_{c}$ decreases, widening the $\hat{\kappa}$-interval for which there exist three stationarypulse solutions: in particular, $\kappa_{c} \rightarrow 0$ as $\sigma \rightarrow 0$. Conversely, as $\sigma$ increases, $\kappa_{c}$ increases toward $\frac{1}{2}$, thus decreasing the size of the three-pulse regime. For $\hat{\kappa}>\frac{1}{2}$, qualitatively, the bifurcation scenario remains unchanged; the effect of increasing $\sigma$ is simply to widen the pulse width $a$. Finally, note that the qualitative behavior of the function $G(a)$, which determines the existence of stationary-pulse solutions, follows from the fact that both $w(x)$ and $I(x)$ are monotonically decreasing functions of $|x|$ and are symmetric about $x=0$.

3.2. Stability analysis. The stability of a stationary pulse of width $a$ is determined by writing $u(x, t)=U(x)+\varphi(x, t)$ and $q(x, t)=Q(x)+\psi(x, t)$ with $Q(x)=U(x)$ and expanding (1.2) to first-order in $(\varphi, \psi)$. This leads to the linear equation

$$
\begin{aligned}
\frac{\partial \varphi(x, t)}{\partial t} & =-\varphi(x, t)+\int_{-\infty}^{\infty} w\left(x-x^{\prime}\right) H^{\prime}\left(U\left(x^{\prime}\right)\right) \varphi\left(x^{\prime}, t\right) d x^{\prime}-\beta \psi(x, t), \\
\frac{1}{\epsilon} \frac{\partial \psi(x, t)}{\partial t} & =-\psi(x, t)+\varphi(x, t) .
\end{aligned}
$$

We assume that $\varphi, \psi \in L^{1}(\mathbf{R})$. The spectrum of the associated linear operator is found by taking $\varphi(x, t)=\mathrm{e}^{\lambda t} \varphi(x)$ and $\psi(x, t)=\mathrm{e}^{\lambda t} \psi(x)$ and using the identity

$$
\frac{d H(U(x))}{d U}=\frac{\delta(x-a)}{\left|U^{\prime}(a)\right|}+\frac{\delta(x+a)}{\left|U^{\prime}(-a)\right|}
$$

where

$$
U^{\prime}(x)=\frac{1}{1+\beta}\left[I^{\prime}(a)+w(x+a)-w(x-a)\right]
$$

and $U^{\prime}(-a)=-U^{\prime}(a)>0$. We then obtain the eigenvalue equation

$$
\left(\lambda+1+\frac{\epsilon \beta}{\lambda+\epsilon}\right) \varphi(x)=\frac{w(x+a)}{\left|U^{\prime}(-a)\right|} \varphi(-a)+\frac{w(x-a)}{\left|U^{\prime}(a)\right|} \varphi(a) .
$$

Note that we have formally differentiated the Heaviside function, which is permissible since it arises inside a convolution. One could also develop the linear stability analysis by considering perturbations of the threshold crossing points along the lines of Amari [1]. Since we are linearizing about a stationary rather than a traveling pulse, we can analyze the spectrum of the linear operator without the recourse to Evans functions. 
There exist three types of solutions to (3.7). The first consists of functions $\varphi(x)$ that vanish at $x= \pm a$ and $\lambda=\lambda_{ \pm}^{(0)}$ with $\lambda_{ \pm}^{(0)}$ given by

$$
\lambda_{ \pm}^{(0)}=\frac{-(1+\epsilon) \pm \sqrt{(1+\epsilon)^{2}-4 \epsilon(1+\beta)}}{2} .
$$

Note that $\lambda_{ \pm}^{(0)}$ belong to the essential spectrum since they have infinite multiplicity and that they are always real and negative. Thus they do not contribute to instabilities. The second consists of solutions of the form $\varphi(x)=A[w(x+a)-w(x-a)]$ with $\lambda$ given by the roots of the equation

$$
\lambda+1+\frac{\epsilon \beta}{\lambda+\epsilon}=\frac{w(0)-w(2 a)}{\left|U^{\prime}(a)\right|} .
$$

It follows that $\lambda=\lambda_{ \pm}$, where

$$
\lambda_{ \pm}=\frac{-\Lambda \pm \sqrt{\Lambda^{2}-4(1-\Gamma) \epsilon(1+\beta)}}{2}
$$

with

$$
\Lambda=1+\epsilon-(1+\beta) \Gamma, \quad \Gamma=\frac{w(0)-w(2 a)}{w(0)-w(2 a)+D},
$$

and $D=\left|I^{\prime}(a)\right|$. Finally, the third type of solution is $\varphi(x)=A[w(x+a)+w(x-a)]$ with $\lambda$ given by the roots of the equation

$$
\lambda+1+\frac{\epsilon \beta}{\lambda+\epsilon}=\frac{w(0)+w(2 a)}{\left|U^{\prime}(a)\right|} .
$$

This yields $\lambda=\widehat{\lambda}_{ \pm}$, where

$$
\widehat{\lambda}_{ \pm}=\frac{-\widehat{\Lambda} \pm \sqrt{\widehat{\Lambda}^{2}-4(1-\widehat{\Gamma}) \epsilon(1+\beta)}}{2}
$$

with

$$
\widehat{\Lambda}=1+\epsilon-(1+\beta) \widehat{\Gamma}, \quad \widehat{\Gamma}=\frac{w(0)+w(2 a)}{w(0)-w(2 a)+D} .
$$

A stationary-pulse solution will be stable provided that $\operatorname{Re} \lambda_{ \pm}, \operatorname{Re} \widehat{\lambda}_{ \pm}<0$.

In the limiting case of a homogeneous input, for which $D=0,(3.10)$ and (3.13) become

$$
\lambda_{-}=0, \quad \lambda_{+}=\beta-\epsilon,
$$

and

$$
\widehat{\lambda}_{ \pm}=\frac{-\widehat{\Lambda}_{0} \pm \sqrt{\widehat{\Lambda}_{0}^{2}+4 \epsilon(1+\beta)\left(\widehat{\Gamma}_{0}-1\right)}}{2}
$$


with

$$
\begin{gathered}
\widehat{\Lambda}_{0}=\epsilon+1-(1+\beta) \widehat{\Gamma}_{0}, \\
\widehat{\Gamma}_{0}=\frac{w(0)+w(2 a)}{w(0)-w(2 a)} .
\end{gathered}
$$

Since $\widehat{\Gamma}_{0}>1$ for finite pulse width $a$, it follows that $\widehat{\lambda}_{+}>0$ for all parameter values, and, hence, a stationary pulse (if it exists) is unstable in the case of the homogeneous network described by (1.2). This result is consistent with Amari's previous analysis [1]. He showed that in order to stabilize a stationary pulse within a homogeneous network, it is necessary to include some form of lateral inhibition. If a weak input inhomogeneity is subsequently introduced into the network, then the peak of the activity profile moves to a local maximum of the input where it is pinned.

For a nonzero Gaussian input, the gradient of a stationary pulse is given by $D(a)$, where

$$
D(a)=\frac{a \mathcal{I}}{\sigma^{2}} \mathrm{e}^{-(a / \sigma)^{2} / 2} .
$$

Using the gradient, we wish to determine the stability of the pulse in terms of the pulse width $a$, with $a=a(\mathcal{I})$ given by one of the solutions of (3.3) for fixed $\kappa, \beta$. Stability of the stationary pulse corresponds to the following conditions:

$$
\Gamma, \hat{\Gamma}<1, \quad \Lambda, \hat{\Lambda}>0 .
$$

However, there are redundancies. First, by inspection of (3.11), the condition $\Gamma<1$ is automatically satisfied. The conditions $\Lambda, \hat{\Lambda}>0$ are equivalent to

$$
\Gamma, \hat{\Gamma}<\frac{1+\epsilon}{1+\beta},
$$

and, since $\Gamma<\hat{\Gamma}$, it follows that the condition on $\Gamma$ is redundant. Hence, stability of the stationary pulse reduces to the conditions

$$
\hat{\Gamma}<1, \quad \hat{\Gamma}<\frac{1+\epsilon}{1+\beta},
$$

in which the latter is redundant for $\epsilon>\beta$, while the former is redundant for $\epsilon<\beta$. These conditions translate in terms of the gradient $D$ as

$$
\begin{array}{ll}
\epsilon>\beta: & D(a)>2 w(2 a) \equiv D_{\mathrm{SN}}(a), \\
\epsilon<\beta: & D(a)>D_{c}(a),
\end{array}
$$

where

$$
D_{c}(a)=2 w(2 a)+\left(\frac{\beta-\epsilon}{1+\epsilon}\right)(w(0)+w(2 a)) .
$$


We now relate stability of the stationary pulse to the gradient $D$ on different branches of the existence curves shown in Figure 2 for $w(x)$ given by the exponential distribution (2.1).

Stability for $\epsilon>\boldsymbol{\beta}$. Equation (3.3) implies that $D(a)=2 w(2 a)-G^{\prime}(a)$. Thus, stability condition (3.20) is satisfied when $G^{\prime}(a)<0$ and not satisfied when $G^{\prime}(a)>0$. Saddle-node bifurcation points occur when $G^{\prime}(a)=0$, i.e., when $D(a)$ passes through $D_{\mathrm{SN}}$, due to the vanishing of a single real eigenvalue $\hat{\lambda}_{+}$. We can make the following conclusions about the solution branches. In scenario (i) there are three solution branches. On the lower and upper solution branches, $G^{\prime}(a)>0$, while $G^{\prime}(a)<0$ on the middle branch, indicating that the former are always unstable and that the latter is stable for $\epsilon>\beta$. In scenario (ii) there are two solution branches: using the same arguments, the lower branch is always unstable while, for $\epsilon>\beta$, the upper branch is stable.

Hopf curves for $\epsilon<\boldsymbol{\beta}$. If $\epsilon<\beta$, then a Hopf bifurcation can occur due to a complex pair of eigenvalues $\hat{\lambda}_{ \pm}$crossing into the right half complex plane. The Hopf bifurcation point is determined by the condition $\hat{\Gamma}=(1+\varepsilon) /(1+\beta)<1$, which is equivalent to the gradient condition $D(a)=D_{c}(a)>D_{\mathrm{SN}}(a)$. It follows that only branches determined to be stable for $\epsilon>\beta$ can undergo a Hopf bifurcation when $\epsilon<\beta$. Moreover, the Hopf bifurcation points coincide with saddle-node bifurcation points precisely at the point $\beta=\epsilon$, where there is a pair of zero eigenvalues suggestive of a codimension 2 Takens-Bogdanov bifurcation. As $\epsilon$ decreases from $\beta$, we expect the Hopf bifurcation point(s) to traverse these previously stable branches from the saddle-node point(s). In order to illustrate this, we find a relationship for $D(a)$ which does not depend explicitly on $\mathcal{I}$. Using (3.3), the input gradient $D$ can be related as

$$
\begin{aligned}
D(a) & =\left|I^{\prime}(a)\right| \\
& =\frac{a}{\sigma^{2}} I(a) \\
& =\frac{a}{\sigma^{2}}(\kappa(1+\beta)-W(2 a)) .
\end{aligned}
$$

We restrict $a$ here depending on which branch of the existence curve we are considering. In each of the scenarios discussed in section 3.1, we examine graphically the crossings of the curves $D(a), D_{c}(a)$ : stability corresponds to $D(a)>D_{c}(a)$ with Hopf points at $D(a)=D_{c}(a)$. Figure 3 illustrates the generic behavior in these scenarios. The left column presents the graphs of $D$ and of $D_{c}$ for different values of $\epsilon$ spanning the interval [0, $\left.\beta\right]$; intersection points indicate Hopf bifurcation points. The right column graphs the corresponding Hopf curves in $(a, \epsilon)$ parameter space. Note that the upper branch in scenario (ii) is always stable for sufficiently large input $\mathcal{I}$, that is, for large pulse width $a$, for (3.3) implies that $I(a) \sim(1+\beta) \kappa-1 / 2$, and hence $D \sim[(1+\beta) \kappa-1 / 2]\left(a / \sigma^{2}\right)$ as $a \rightarrow \infty$. Since $\widehat{D}_{c}(a) \rightarrow(\beta-\epsilon) /(1+\epsilon)$ and $\mathrm{e}^{-2 a} \rightarrow 0$ as $a \rightarrow \infty$, it follows that both stability conditions (3.20) and (3.21) are satisfied in this limit. Varying $\sigma$ does not affect the qualitative behavior of the Hopf bifurcation curves. Since $\sigma$ appears only in (3.23), the effect of increasing $\sigma$ is to shrink the graph of $D$ by a factor $1 / \sigma^{2}$, causing the Hopf curves in the right column of Figure 3 to be stretched downward, thus increasing the size of the stability region in the $(a, \epsilon)$-plane.

4. Numerical results. In our numerical simulations we use a Runge-Kutta (RK4) scheme with 4000-10000 spatial grid points and time step $d t=0.02$, evaluating the integral term by 


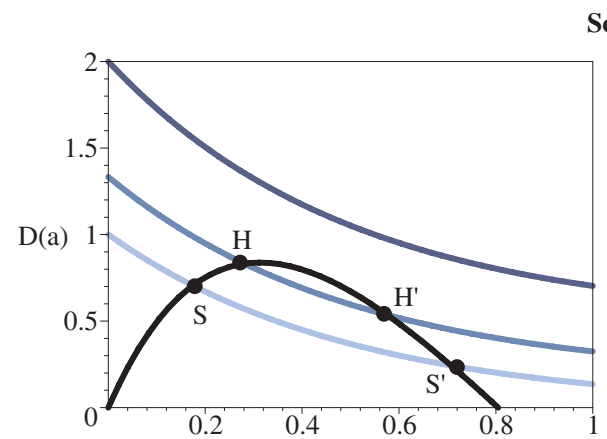

Scenario (i)
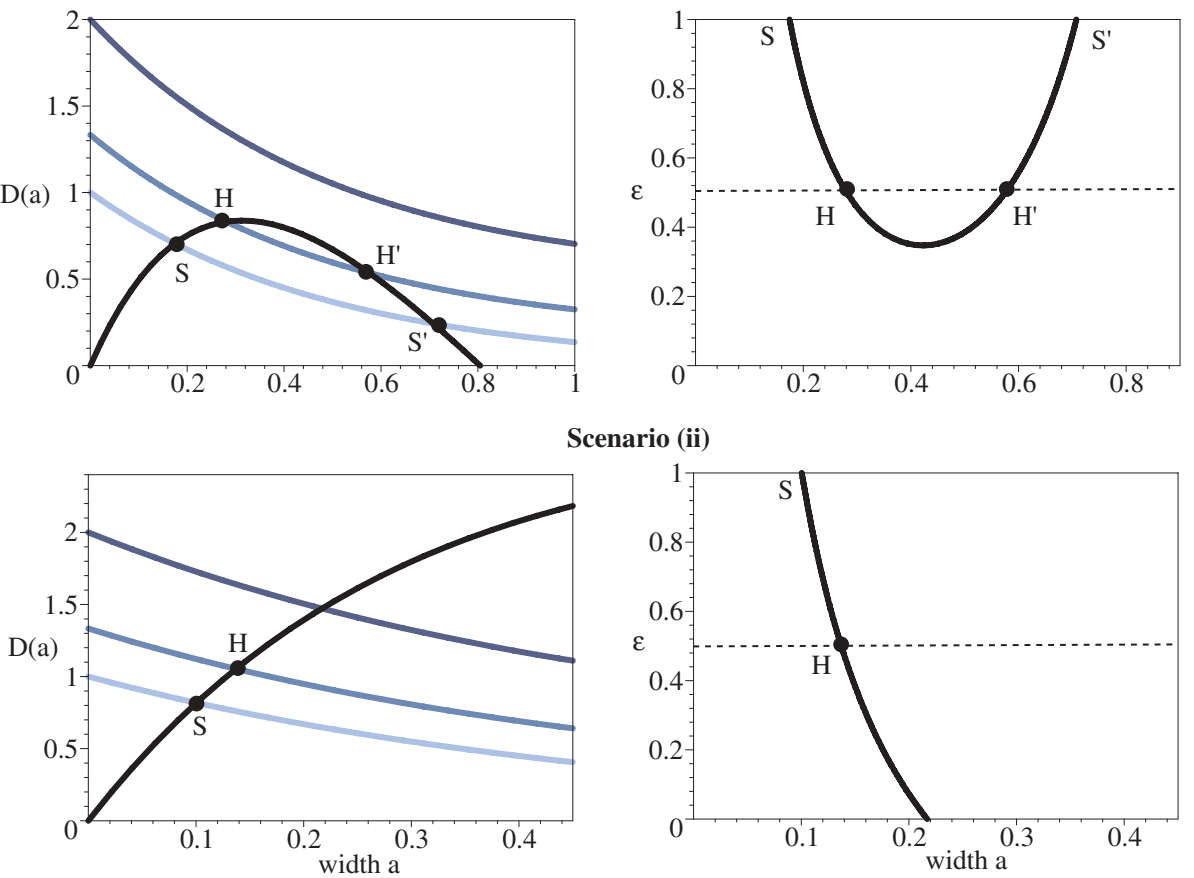

Scenario (ii)

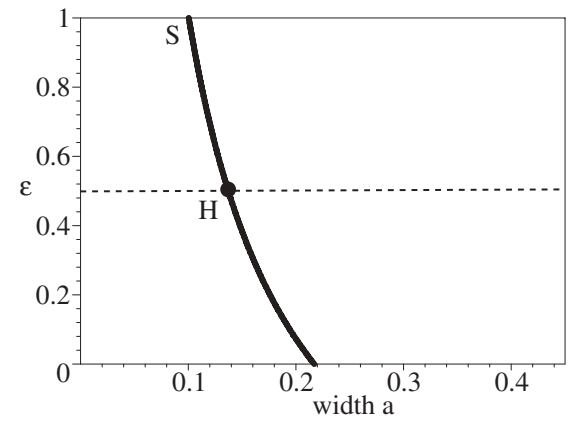

Figure 3. Left column: Gradient curves for the two bifurcation scenarios shown in Figure 2: (i) $\kappa_{c}<\hat{\kappa}<\frac{1}{2}$ and (ii) $\hat{\kappa}>\frac{1}{2}$. The thick solid curve shows the input gradient $D(a)$ as a function of pulse width a. The lighter curves show the critical gradient $D_{c}(a)$ as function of a for $\epsilon=0.0,0.5,1.0$ and $\beta=1$. For a given value of $\epsilon<\beta$, a stationary pulse of width a is stable provided that $D(a)>D_{c}(a)$. A pulse loses stability via a Hopf bifurcation at any intersection points $D(a)=D_{c}(a)$. The Hopf bifurcation point $(s)$ for $\epsilon=0.5$ are indicated by $H, H^{\prime}$. In the limit $\epsilon \rightarrow \beta$, we have $H, H^{\prime} \rightarrow S, S^{\prime}$. Right column: Corresponding Hopf stability curves in the $(a, \epsilon)$-plane.

quadrature. Boundary points freely evolve according to the scheme rather than by prescription, and the size of the domain is chosen so that the stationary pulse is unaffected by the boundaries.

4.1. Hopf bifurcation to a breather. Numerically solving the one-dimensional rate equation (1.2), we find that the Hopf instability of the upper solution branch in bifurcation scenario (ii) induces a breather-like oscillatory pulse solution; see Figures 4 and 5 . As the input amplitude $\mathcal{I}$ is slowly reduced below $\mathcal{I}_{H B}$, the oscillations steadily grow until a new instability point is reached. Interestingly, the breather persists over a range of inputs beyond this secondary instability, except that it now periodically emits pairs of traveling pulses, as illustrated in Figure 6. In fact, such a solution is capable of persisting even when the input is below threshold, that is, for $\mathcal{I}<(1+\beta) \kappa$. Note that although the homogeneous network $(\mathcal{I}=0)$ also supports the propagation of traveling pulses, it does not support the existence of a breather that can act as a source of these waves.

Our simulations suggest both supercritical and subcritical Hopf bifurcations can occur for scenario (ii). The conclusion of supercriticality is based on the evidence that there is continuous growth of the amplitude of the oscillations from the stationary solution as $\mathcal{I}$ is 


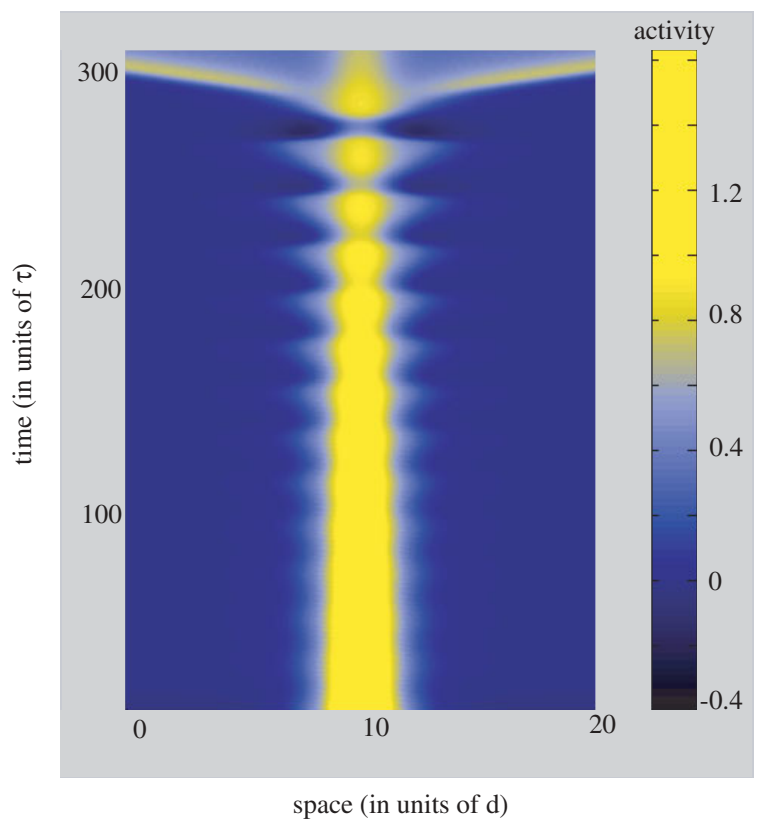

Figure 4. Breather-like solution arising from a Hopf instability of a stationary pulse due to a slow reduction in the amplitude $\mathcal{I}$ of the Gaussian input inhomogeneity (3.1) for an exponential weight distribution. Here $\mathcal{I}=5.5$ at $t=0$ and $\mathcal{I}=1.5$ at $t=250$. Other parameter values are $\epsilon=0.03, \beta=2.5, \kappa=0.3, \sigma=1.0$. The amplitude of the oscillation steadily grows until it undergoes a secondary instability at $\mathcal{I} \approx 2$, beyond which the breather persists and periodically generates pairs of traveling pulses (only one of which is shown). The breather itself disappears when $\mathcal{I} \approx 1$.

reduced through the predicted bifurcation point, and, moreover, that the frequency of the oscillatory solution near the bifurcation point is approximately equal to the predicted Hopf frequency

$$
\omega_{H}=\operatorname{Im} \hat{\lambda}_{ \pm}=\sqrt{\epsilon(\beta-\epsilon)} .
$$

For example, the Hopf bifurcation of the stationary pulse for the parameter values given in Figure 4 was determined numerically to be supercritical. Conversely, the Hopf bifurcation in scenario (i) appears to be subcritical. Furthermore, the basin of attraction of the stable pulse on the middle branch seems to be small, rendering it, as well as any potential breather, difficult to approach. Hence, we did not investigate this case further.

As mentioned above, a secondary instability occurs at some $\mathcal{I}<\mathcal{I}_{H B}$, whereupon traveling pulses are emitted: this behavior appears to occur only for values of $\epsilon$ that support traveling pulses in the homogeneous model $(\mathcal{I}=0)$. As the point of secondary instability is approached, the breather starts to exhibit behavior suggestive of pulse emission, except that the recovery variable $q$ increases rapidly enough to prevent the nascent waves from propagating. On the other hand, beyond the point of instability, recovery is not fast enough to block pulse emission; we also find that the activity variable $u$ always drops well below threshold after each emission. Interestingly, for a range of input amplitudes we observe frequency-locking between the oscillations of the breather and the rate at which pairs of pulses are emitted from the 

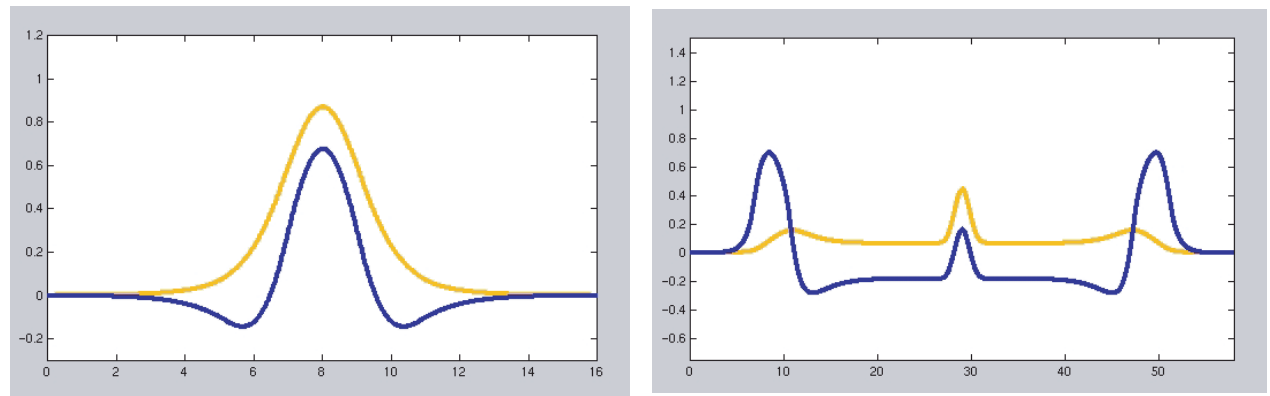

Figure 5. Snapshots of the oscillatory behavior of (a) the breathing pulse $(\mathcal{I}=2.2)$ and (b) the pulse emitter $(\mathcal{I}=1.3)$ far beyond the bifurcation point. The graphs of $U$ and $Q$ are indicated in blue and gold, respectively, with the horizontal axis representing space in units of $d$, the spatial extent associated with the exponential weight function. Other parameters are $\beta=2.5, \kappa=0.3, \epsilon=0.03$. Clicking on the above images displays the associated movies.
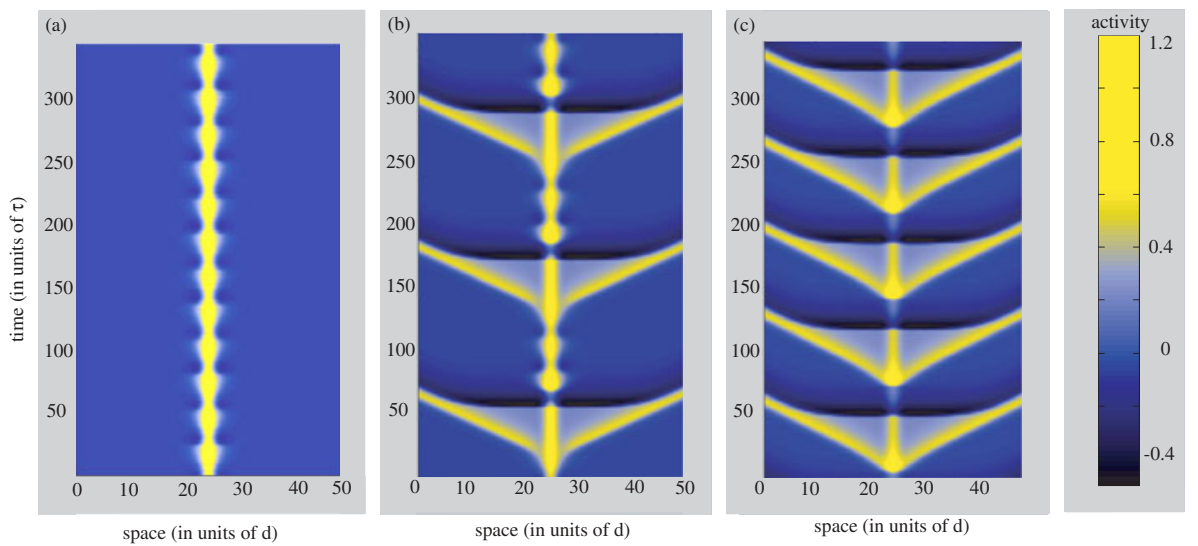

Figure 6. Mode-locking in the transition from breather to pulse emitter. (a) 0:1 mode-locking for $\mathcal{I}=2.3$, (b) 1:4 mode-locking for $\mathcal{I}=2.1$, (c) 1:2 mode-locking for $\mathcal{I}=1.3$.

breather. Two examples of $n: m$ mode-locked solutions are shown in Figure 6 , in which there are $n$ pairs of pulses emitted per $m$ oscillation cycles of the central breather. As $\mathcal{I}$ is reduced further, the only mode that is seen is $1: 2$, which itself ultimately vanishes, and the system is attracted to the subthreshold solution.

4.2. Breathers in a biophysical model. Although the rate model is very useful as an analytically tractable model of neural tissue, it is important to determine whether or not its predictions regarding spatio-temporal dynamics hold in more biophysically realistic conductancebased models. For concreteness, we consider a version of the Traub model, in which there is an additional slow potassium M-current that produces the effect of spike-rate adaptation [9]. We also discretize space by setting $x=j \Delta x$ for $j=1, \ldots, N$ and label neurons by the index $j$. The membrane potential of the $j$ th neuron satisfies the following Hodgkin-Huxley-like 
dynamics [9]:

$$
C \frac{d V_{j}}{d t}=-I_{\mathrm{ion}}\left(V_{j}, m, n, h, q\right)-I_{j}^{\mathrm{syn}}(t)+I_{j}
$$

with synaptic current

$$
\begin{aligned}
I_{j}^{\mathrm{syn}}(t) & =g_{\mathrm{syn}} \sum_{k} w(|j-k|) s_{k}(t)\left(V-V_{\mathrm{syn}}\right), \\
\frac{d s_{j}}{d t} & =K\left(V_{j}(t)\right)\left(1-s_{j}\right)-\frac{1}{\tau} s_{j}
\end{aligned}
$$

and ionic currents

$$
\begin{gathered}
I_{\text {ion }}(V, m, n, h)=g_{\mathrm{L}}\left(V-V_{\mathrm{L}}\right)+g_{\mathrm{K}} n^{4}\left(V-V_{\mathrm{K}}\right)+g_{\mathrm{Na}} m^{3} h\left(V-V_{\mathrm{Na}}\right)+g_{q} q\left(V-V_{\mathrm{K}}\right), \\
\tau_{p}(V) \frac{d p}{d t}=p_{\infty}(V)-p, \quad p \in\{m, n, h, q\} .
\end{gathered}
$$

The various biophysical model functions and the parameters used in the numerics are listed in Appendix A. Note that we have also included an external Gaussian input current $I_{j}$ in order to investigate the behavior predicted by the rate model. Without this external input, the biophysical model has previously been shown to support a traveling pulse, consisting of either a single action-potential or a packet of action-potentials [9]. Since the firing rate model describes the average activity, we interpret high activity as repetitive firing of neurons and low activity as neurons that are subthreshold or quiescent. Hence, we expect that the application of a strong unimodal input should generate a stationary pulse, i.e., a localized region of neurons that are repetitively firing, surrounded by a region of neurons that are quiescent. Subsequent reduction of the input should lead to oscillations in this localized region followed by emission of action-potentials or packets of action-potentials.

One obvious difference between this biophysical model and the rate model discussed in section 3 is that the gating variable associated with spike-rate adaptation evolves according to more complicated nonlinear dynamics, while that of the firing rate model evolves according to simple linear dynamics. Nevertheless, the behavior of the rate model appears to carry over to the biophysical model, thus lending support to the ability of rate models to describe the averaged behavior of spiking biophysical models. For large input amplitude $\mathcal{I}$, the system approaches a solution in which a region, localized about the input, is repetitively firing, while the outer region is quiescent; moreover, the firing rate is maximal in the center of this region and decreases toward the boundaries, which is analogous to the stationary pulse of the firing rate model. As $\mathcal{I}$ is subsequently decreased, there is a transition to breather-like behavior: periodically, packets or bursts of action-potentials begin to propagate from the active region and, shortly thereafter, fail to propagate as the newly excited region recovers. As in the rate model, further reduction of the amplitude $\mathcal{I}$ leads to a transition to a state in which packets of persistent action-potentials are emitted. Two examples are shown in Figure 7. The first is in a regime where the breather still dominates with the occasional emission of wave packets. The second corresponds to regular pulse emission, in which periodic bursts of persistent actionpotentials are emitted, each followed by an interlude of subthreshold behavior in the vicinity 

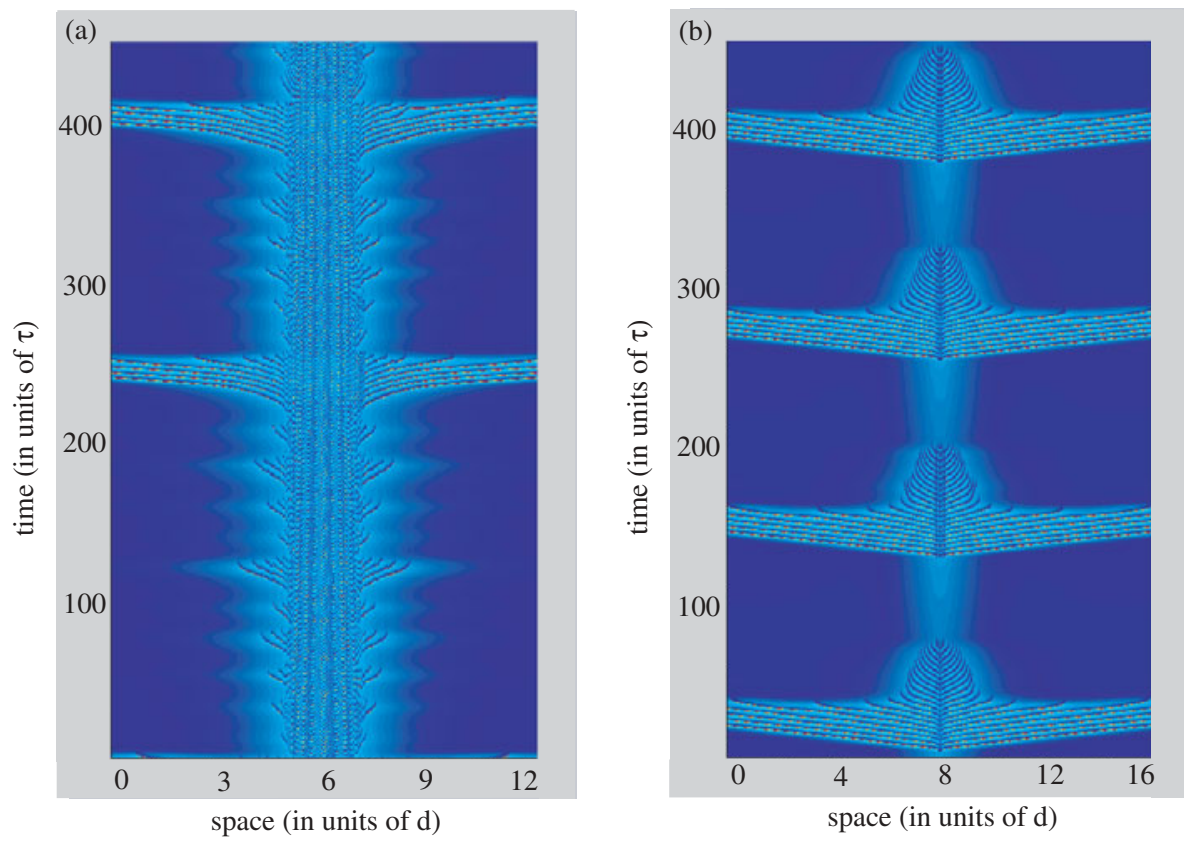

Figure 7. Breathers in a biophysical model with an exponential weight distribution. (a) $\mathcal{I}=75 \mathrm{~mA} / \mathrm{cm}^{2}$, (b) $\mathcal{I}=50 \mathrm{~mA} / \mathrm{cm}^{2}$, where $\mathcal{I}$ is the amplitude of the Gaussian input. Other parameter values are specified in Appendix A.

of the input; this is similar to the 1:2 pulse emitter of the firing rate model shown in Figure 6. We expect similar behavior to occur in other biophysical models that have some form of sufficiently slow negative feedback.

5. Two-dimensional pulses. We now extend our analysis to derive conditions for the existence and stability of radially symmetric stationary-pulse solutions of a two-dimensional version of (1.2):

$$
\begin{aligned}
\frac{\partial u(\mathbf{r}, t)}{\partial t} & =-u(\mathbf{r}, t)+\int_{\mathbb{R}^{2}} w\left(\left|\mathbf{r}-\mathbf{r}^{\prime}\right|\right) H\left(u\left(\mathbf{r}^{\prime}, t\right)-\kappa\right) d \mathbf{r}^{\prime}-\beta q(\mathbf{r}, t)+I(r), \\
\frac{1}{\epsilon} \frac{\partial q(\mathbf{r}, t)}{\partial t} & =-q(\mathbf{r}, t)+u(\mathbf{r}, t)
\end{aligned}
$$

where $\mathbf{r}=(r, \theta)$ and $\mathbf{r}^{\prime}=\left(r^{\prime}, \theta^{\prime}\right)$. Both the input $I(r)$ and the weight distribution $w(r)$ are taken to be positive monotonically decreasing functions in $L^{1}\left(\mathbf{R}^{+}\right)$. As in the one-dimensional case, stationary-pulse solutions are unstable in a homogeneous excitatory network but can be stabilized by the local input. Our analysis should be contrasted with a number of recent studies of two-dimensional stationary pulses [32, 34, 20]. These latter studies consider homogeneous networks with uniform external inputs and include both excitatory and inhibitory synaptic coupling. Thus the inhibition is nonlocal rather than local as in our model.

5.1. Stationary-pulse existence. We begin by developing a formal representation of the two-dimensional stationary-pulse solution for a general monotonically decreasing weight func- 
tion $w$. We then generate stationary-pulse existence curves for the specific case of an exponential weight function and analyze their dependence on the parameters of the system. Since we cannot obtain a closed form for the solution in the case of the exponential weight distribution, we also derive an explicit solution for the case of a modified Bessel weight function that approximates the exponential. For concreteness, we consider a Gaussian input $I(r)=\mathcal{I}^{-r^{2} / 2 \sigma^{2}}$.

Pulse construction for a general synaptic weight function. A radially symmetric stationary-pulse solution of (5.1) is $u=q=U(r)$ with $U$ depending only upon the spatial variable $r$ such that

$$
\begin{array}{lll}
U(r)>\kappa, \quad r \in(0, a) ; & & U(\infty)=0 \\
U(a)=\kappa ; & & U(0)<\infty \\
U(r)<\kappa, \quad r \in(a, \infty) . &
\end{array}
$$

Substituting into (5.1) gives

$$
(1+\beta) U(r)=M(a, r)+I(r)
$$

where

$$
\begin{aligned}
M(a, r) & =\int_{\mathbf{R}^{2}} w\left(\left|\mathbf{r}-\mathbf{r}^{\prime}\right|\right) H\left(U\left(r^{\prime}\right)-\kappa\right) d \mathbf{r}^{\prime} \\
& =\int_{0}^{2 \pi} \int_{0}^{a} w\left(\left|\mathbf{r}-\mathbf{r}^{\prime}\right|\right) r^{\prime} d r^{\prime} d \theta .
\end{aligned}
$$

In order to calculate the double integral in (5.3) we use the Fourier transform, which for radially symmetric functions reduces to a Hankel transform. To see this, consider the twodimensional Fourier transform of the radially symmetric weight function $w$, expressed in polar coordinates,

$$
\begin{aligned}
w(r) & =\frac{1}{2 \pi} \int_{\mathbb{R}^{2}} e^{i(\mathbf{r} \cdot \mathbf{k})} \breve{w}(\mathbf{k}) d \mathbf{k} \\
& =\frac{1}{2 \pi} \int_{0}^{\infty}\left(\int_{0}^{2 \pi} e^{i r \rho \cos (\theta-\phi)} \breve{w}(\rho) d \phi\right) \rho d \rho,
\end{aligned}
$$

where $\breve{w}$ denotes the Fourier transform of $w$ and $\mathbf{k}=(\rho, \phi)$. Using the integral representation

$$
\frac{1}{2 \pi} \int_{0}^{2 \pi} e^{i r \rho \cos (\theta-\varphi)} d \theta=J_{0}(r \rho)
$$

where $J_{\nu}(z)$ is the Bessel function of the first kind, we express $w$ in terms of its Hankel transform of order zero,

$$
w(r)=\int_{0}^{\infty} \breve{w}(\rho) J_{0}(r \rho) \rho d \rho
$$

which, when substituted into (5.3), gives

$$
M(a, r)=\int_{0}^{2 \pi} \int_{0}^{a}\left(\int_{0}^{\infty} \breve{w}(\rho) J_{0}\left(\rho\left|\mathbf{r}-\mathbf{r}^{\prime}\right|\right) \rho d \rho\right) r^{\prime} d r^{\prime} d \theta^{\prime} .
$$


Switching the order of integration gives

$$
M(a, r)=\int_{0}^{\infty} \breve{w}(\rho)\left(\int_{0}^{2 \pi} \int_{0}^{a} J_{0}\left(\rho\left|\mathbf{r}-\mathbf{r}^{\prime}\right|\right) r^{\prime} d r^{\prime} d \theta^{\prime}\right) \rho d \rho
$$

In polar coordinates $\left|\mathbf{r}-\mathbf{r}^{\prime}\right|=\sqrt{r^{2}+r^{\prime 2}-2 r r^{\prime} \cos \left(\theta-\theta^{\prime}\right)}$,

$$
\begin{aligned}
\int_{0}^{2 \pi} \int_{0}^{a} J_{0}\left(\rho\left|\mathbf{r}-\mathbf{r}^{\prime}\right|\right) r^{\prime} d r^{\prime} d \theta^{\prime} & =\int_{0}^{2 \pi} \int_{0}^{a} J_{0}\left(\rho \sqrt{r^{2}+r^{\prime 2}-2 r r^{\prime} \cos \left(\theta-\theta^{\prime}\right)}\right) r^{\prime} d r^{\prime} d \theta^{\prime} \\
& =\frac{1}{\rho^{2}} \int_{0}^{2 \pi} \int_{0}^{a \rho} J_{0}\left(\sqrt{R^{2}+R^{\prime 2}-2 R R^{\prime} \cos \left(\theta^{\prime}\right)}\right) R^{\prime} d R^{\prime} d \theta^{\prime}
\end{aligned}
$$

where $R=r \rho$ and $R^{\prime}=r^{\prime} \rho$. To separate variables, we use the addition theorem

$$
J_{0}\left(\sqrt{R^{2}+R^{\prime 2}-2 R R^{\prime} \cos \theta^{\prime}}\right)=\sum_{m=0}^{\infty} \epsilon_{m} J_{m}(R) J_{m}\left(R^{\prime}\right) \cos m \theta^{\prime},
$$

where $\epsilon_{0}=1$ and $\epsilon_{n}=2$ for $n \geq 1$. Since $\int_{0}^{2 \pi} \cos m \theta^{\prime} d \theta^{\prime}=0$ for $m \geq 1$, it follows that

$$
\begin{aligned}
\int_{0}^{2 \pi} \int_{0}^{a} J_{0}\left(\rho\left|\mathbf{r}-\mathbf{r}^{\prime}\right|\right) r^{\prime} d r^{\prime} d \theta^{\prime} & =\frac{1}{\rho^{2}} \sum_{m=0}^{\infty} \epsilon_{m} J_{m}(R) \int_{0}^{a \rho} J_{m}\left(R^{\prime}\right) R^{\prime} d R^{\prime} \int_{0}^{2 \pi} \cos m \theta^{\prime} d \theta^{\prime} \\
& =\frac{2 \pi}{\rho^{2}} J_{0}(R) \int_{0}^{a \rho} J_{0}\left(R^{\prime}\right) R^{\prime} d R^{\prime} \\
& =\frac{2 \pi a}{\rho} J_{0}(r \rho) J_{1}(a \rho) .
\end{aligned}
$$

Hence for general weight $w, M(a, r)$ has the formal representation

$$
M(a, r)=2 \pi a \int_{0}^{\infty} \breve{w}(\rho) J_{0}(r \rho) J_{1}(a \rho) d \rho .
$$

We now wish to show that for a general monotonically decreasing weight function $w(r)$, the function $M(a, r)$ is necessarily a monotonically decreasing function of $r$. This will ensure that the radially symmetric stationary-pulse solution (5.2) is also a monotonically decreasing function of $r$ in the case of a Gaussian input. Differentiating $M$ with respect to $r$ using (5.3) yields

$$
\frac{\partial M}{\partial r}(a, r)=\int_{0}^{2 \pi} \int_{0}^{a} w^{\prime}\left(\left|\mathbf{r}-\mathbf{r}^{\prime}\right|\right)\left(\frac{r-r^{\prime} \cos \left(\theta^{\prime}\right)}{\sqrt{r^{2}+r^{\prime 2}-2 r r^{\prime} \cos \left(\theta^{\prime}\right)}}\right) r^{\prime} d r^{\prime} d \theta^{\prime} .
$$

By inspection of (5.7), $\frac{\partial M}{\partial r}(a, r)<0$ for $r>a$, since $w^{\prime}(z)<0$. To see that it is also negative for $r<a$ and, thus, monotonic, we instead consider the equivalent Hankel representation of (5.6). Differentiation of $M$ in this case yields

$$
\partial_{2} M(a, r) \equiv \frac{\partial M}{\partial r}(a, r)=-2 \pi a \int_{0}^{\infty} \rho \breve{w}(\rho) J_{1}(r \rho) J_{1}(a \rho) d \rho
$$


implying that

$$
\operatorname{sgn}\left(\partial_{2} M(a, r)\right)=\operatorname{sgn}\left(\partial_{2} M(r, a)\right)
$$

Consequently $\frac{\partial M}{\partial r}(a, r)<0$ also for $r<a$. Hence $U$ is monotonically decreasing in $r$ for any monotonic synaptic weight function $w$.

Exponential weight function. Consider the radially symmetric exponential weight function and its Hankel representation

$$
w(r)=\frac{1}{2 \pi} e^{-r}, \quad \breve{w}(r)=\frac{1}{2 \pi} \frac{1}{\left(1+\rho^{2}\right)^{\frac{3}{2}}} .
$$

The condition for the existence of a stationary pulse is then given by

$$
(1+\beta) \kappa=\mathbf{M}(a)+I(a) \equiv G(a),
$$

where

$$
\mathbf{M}(a) \equiv M(a, a)=a \int_{0}^{\infty} \frac{1}{\left(\rho^{2}+1\right)^{\frac{3}{2}}} J_{0}(a \rho) J_{1}(a \rho) d \rho .
$$

The function $G(a)$ is plotted in Figure 8 for a range of input amplitudes $\mathcal{I}$, with horizontal lines indicating different values of $\hat{\kappa}$; intersection points determine the existence of stationary pulse solutions. Note that the integral expression on the right-hand side of (5.11) can be evaluated explicitly in terms of finite sums of modified Bessel and Struve functions; see Appendix B.

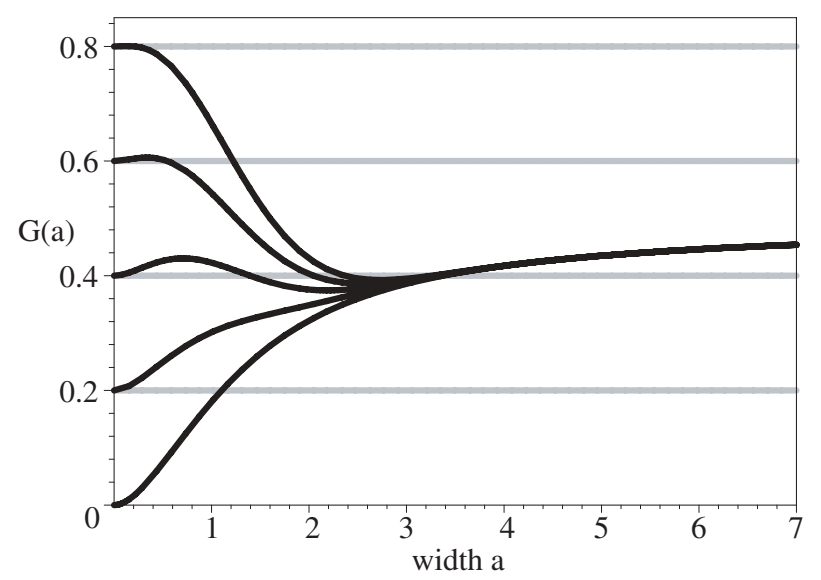

Figure 8. Plot of $G(a)$ defined in (5.10) as a function of pulse width a for various values of input amplitude $\mathcal{I}$ and for fixed input width $\sigma=1$.

We proceed in the same fashion as in the one-dimensional case and generate stationarypulse existence curves for the exponential weight function. Qualitatively the catalogue of bifurcation scenarios is similar, although there is now an additional case. In one dimension we have $G^{\prime}(0)>0$ so that there are always at least two solution branches when $\hat{\kappa}>1 / 2$. On the other hand, in two dimensions we have $G^{\prime}(0)<0$ for sufficiently large input amplitude $\mathcal{I}$ so that it is possible to find only one solution branch for large $\hat{\kappa}$, that is, when $\hat{\kappa}>\kappa_{0}$ for some critical value $\kappa_{0}>1 / 2$. Hence, there are three distinct cases as shown in Figure 9. The effect of varying $\sigma$ identically follows the one-dimensional case. 

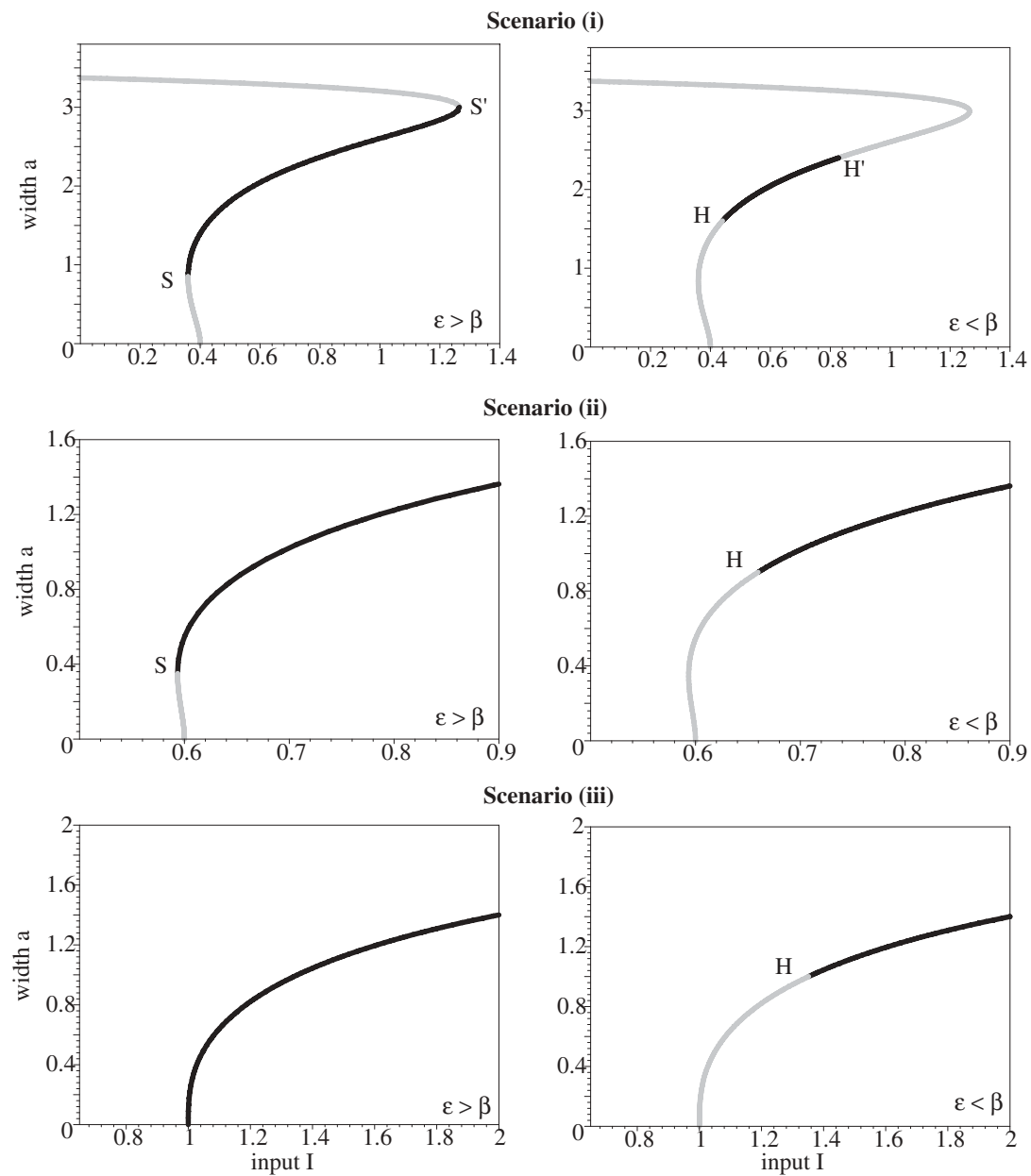

Figure 9. Two-dimensional stationary-pulse existence curves for an exponential weight distribution: (i) $\kappa_{c}<\hat{\kappa}<\frac{1}{2}$, (ii) $\frac{1}{2}<\hat{\kappa}<\kappa_{0}$, and (iii) $\kappa_{0}<\hat{\kappa}$. Other parameter values are $\beta=1, \sigma=1.0$. Black indicates stability, whereas gray indicates instability of the stationary pulse. Saddle-node bifurcation points are indicated by $S, S^{\prime}$ and Hopf bifurcation points by $H, H^{\prime}$.

Modified Bessel weight function. In the case of the exponential weight function $w$ we do not have a closed form for the integral in (5.6). Here we consider a nearby problem where we are able to construct the stationary-pulse solution explicitly. Consider the radially symmetric weight function, normalized to unity,

$$
w(r)=\frac{2}{3 \pi}\left(K_{0}(r)-K_{0}(2 r)\right),
$$

where $K_{\nu}$ is the modified Bessel function of the second kind, whose Hankel transform is

$$
\breve{w}(r)=\frac{2}{3 \pi}\left(\frac{1}{\rho^{2}+1}-\frac{1}{\rho^{2}+2^{2}}\right) .
$$

The coefficient $2 / 3 \pi$ is chosen so that there is a good fit with the exponential distribution as 
shown in Figure 10(a). Note that

$$
w(0)=\frac{1}{2 \pi} \frac{4 \ln (2)}{3} \approx \frac{1}{2 \pi}(0.924), \quad w(r) \sim \frac{1}{3}\left(\frac{\sqrt{2} e^{-r}-e^{-2 r}}{\sqrt{r}}\right) \quad \text { for large } r .
$$

Substituting (5.13) into (5.3), we can explicitly compute the resulting integral using Bessel functions:

$$
a \int_{0}^{\infty} \frac{1}{\rho^{2}+s^{2}} J_{0}(r \rho) J_{1}(a \rho) d \rho=\left\{\begin{array}{cl}
\frac{a}{s} I_{1}(s a) K_{0}(s r) & \text { for } r \geq a \\
\frac{1}{s^{2}}-\frac{a}{s} I_{0}(s r) K_{1}(s a) & \text { for } r<a
\end{array}\right.
$$

where $I_{\nu}$ is the modified Bessel function of the first kind. Substituting into (5.3) shows that

$$
\begin{aligned}
M(a, r) & =2 \pi a \int_{0}^{\infty} \breve{w}(\rho) J_{0}(r \rho) J_{1}(a \rho) d \rho \\
& =\frac{4}{3} a \int_{0}^{\infty}\left(\frac{1}{\rho^{2}+1}-\frac{1}{\rho^{2}+2^{2}}\right) J_{0}(r \rho) J_{1}(a \rho) d \rho \\
& =\left\{\begin{array}{cl}
\frac{4}{3}\left(a I_{1}(a) K_{0}(r)-\frac{a}{2} I_{1}(2 a) K_{0}(2 r)\right) & \text { for } r \geq a, \\
1-\frac{4}{3}\left(a I_{0}(r) K_{1}(a)+\frac{a}{2} I_{0}(2 r) K_{1}(2 a)\right) & \text { for } r<a .
\end{array}\right.
\end{aligned}
$$

The condition for the existence of a stationary pulse of radius $a$ is thus given by (5.10) with

$$
\mathbf{M}(a)=\frac{4}{3}\left(a I_{1}(a) K_{0}(a)-\frac{a}{2} I_{1}(2 a) K_{0}(2 a)\right) .
$$

An example of an exact pulse solution is shown in Figure 10(b).
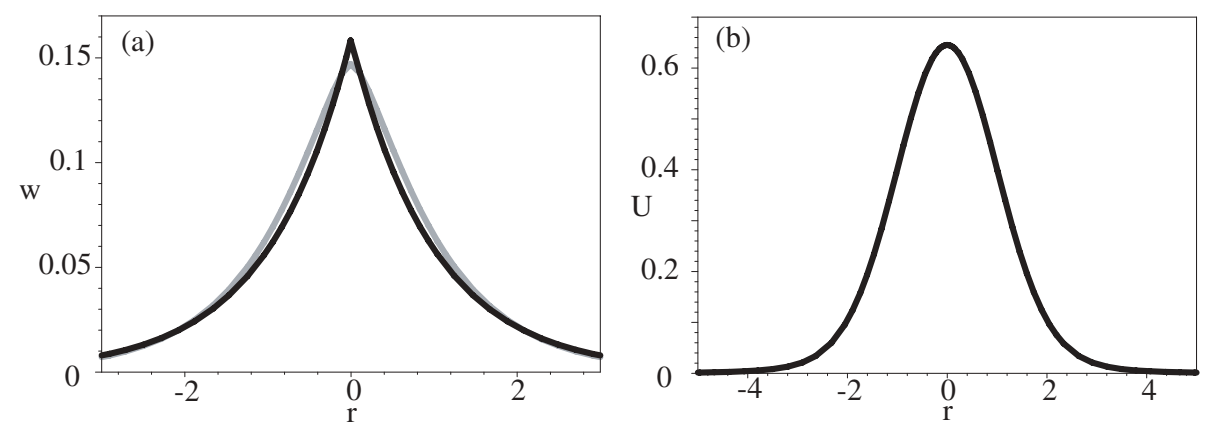

Figure 10. (a) Synaptic weight functions, exponential weight in black and modified Bessel weight in gray. (b) Stationary-pulse solution with half-width, $a=1$, generated by the modified Bessel weight function with $\kappa=0.4, \beta=1, \mathcal{I}=1$.

5.2. Stability analysis. We now analyze the evolution of small time-dependent perturbations of the stationary-pulse solution through linear stability analysis. We investigate saddlenode and Hopf bifurcations of the stationary pulse by relating the eigenvalues to the gradient of the Gaussian input $I$. The behavior of the system near and beyond the Hopf bifurcation is then studied numerically as in one dimension. 
Spectral analysis of the linearized operator. Equation (5.1) is linearized about the stationary solution $(U, Q)$ by introducing the time-dependent perturbations

$$
\begin{aligned}
& u(\mathbf{r}, t)=U(r)+\varphi(\mathbf{r}, t), \\
& q(\mathbf{r}, t)=Q(r)+\psi(\mathbf{r}, t)
\end{aligned}
$$

with $Q=U$ and expanding to first-order in $\varphi, \psi$. This leads to the linearized system of equations

$$
\begin{aligned}
\frac{\partial \varphi}{\partial t}(\mathbf{r}, t) & =-\varphi(\mathbf{r}, t)+\int_{\mathbb{R}^{2}} w\left(\left|\mathbf{r}-\mathbf{r}^{\prime}\right|\right) H^{\prime}\left(U\left(r^{\prime}\right)-\kappa\right) \varphi\left(\mathbf{r}^{\prime}, t\right) d \mathbf{r}^{\prime}-\beta \psi(\mathbf{r}, t), \\
\frac{1}{\epsilon} \frac{\partial \psi}{\partial t}(\mathbf{r}, t) & =-\psi(\mathbf{r}, t)+\varphi(\mathbf{r}, t) .
\end{aligned}
$$

We separate variables

$$
\begin{aligned}
& \varphi(\mathbf{r}, t)=\varphi(\mathbf{r}) e^{\lambda t} \\
& \psi(\mathbf{r}, t)=\psi(\mathbf{r}) e^{\lambda t}
\end{aligned}
$$

to obtain the system

$$
\begin{aligned}
& \lambda \varphi(\mathbf{r})=-\varphi(\mathbf{r})+\int_{\mathbb{R}^{2}} w\left(\left|\mathbf{r}-\mathbf{r}^{\prime}\right|\right) H^{\prime}\left(U\left(r^{\prime}\right)-\kappa\right) \varphi\left(\mathbf{r}^{\prime}\right) d \mathbf{r}^{\prime}-\beta \psi(\mathbf{r}), \\
& \frac{\lambda}{\epsilon} \psi(\mathbf{r})=-\psi(\mathbf{r})+\varphi(\mathbf{r}) .
\end{aligned}
$$

Solving (5.15), we find

$$
\left(\lambda+1+\frac{\beta \epsilon}{\lambda+\epsilon}\right) \varphi(\mathbf{r})=\int_{\mathbb{R}^{2}} w\left(\left|\mathbf{r}-\mathbf{r}^{\prime}\right|\right) H^{\prime}\left(U\left(r^{\prime}\right)-\kappa\right) \varphi\left(\mathbf{r}^{\prime}\right) d \mathbf{r}^{\prime} .
$$

Introducing polar coordinates $\mathbf{r}=(r, \theta)$ and using the result

$$
H^{\prime}(U(r)-\kappa)=\delta(U(r)-\kappa)=\frac{\delta(r-a)}{\left|U^{\prime}(a)\right|},
$$

we obtain

$$
\begin{aligned}
\left(\lambda+1+\frac{\epsilon \beta}{\lambda+\epsilon}\right) \varphi(\mathbf{r}) & =\int_{0}^{2 \pi} \int_{0}^{\infty} w\left(\left|\mathbf{r}-\mathbf{r}^{\prime}\right|\right) \frac{\delta\left(r^{\prime}-a\right)}{\left|U^{\prime}(a)\right|} \varphi\left(\mathbf{r}^{\prime}\right) r^{\prime} d r^{\prime} d \theta^{\prime} \\
& =\frac{a}{\left|U^{\prime}(a)\right|} \int_{0}^{2 \pi} w\left(\left|\mathbf{r}-\mathbf{a}^{\prime}\right|\right) \varphi\left(a, \theta^{\prime}\right) d \theta^{\prime}
\end{aligned}
$$

where $\mathbf{a}^{\prime}=\left(a, \theta^{\prime}\right)$

We consider the following two cases. (i) The function $\varphi$ satisfies the condition

$$
\int_{0}^{2 \pi} w\left(\left|\mathbf{r}-\mathbf{a}^{\prime}\right|\right) \varphi\left(a, \theta^{\prime}\right) d \theta^{\prime}=0
$$


for all $\mathbf{r}$. The integral equation reduces to

$$
\lambda+1+\frac{\beta \epsilon}{\lambda+\epsilon}=0,
$$

yielding the eigenvalues

$$
\lambda_{ \pm}^{(0)}=\frac{-(1+\epsilon) \pm \sqrt{(1+\epsilon)^{2}-4 \epsilon(1+\beta)}}{2} .
$$

This is part of the essential spectrum and is identical to the one-dimensional case: it is negative and does not cause instability. (ii) $\varphi$ does not satisfy the above condition, and we must study the solutions of the integral equation

$$
\mu \varphi(r, \theta)=a \int_{0}^{2 \pi} \mathcal{W}\left(a, r ; \theta-\theta^{\prime}\right) \varphi\left(a, \theta^{\prime}\right) d \theta^{\prime}
$$

where

$$
\lambda+1+\frac{\epsilon \beta}{\lambda+\epsilon}=\frac{\mu}{\left|U^{\prime}(a)\right|}
$$

and $\mathcal{W}(a, r ; \phi)=w\left(\sqrt{r^{2}+a^{2}-2 r a \cos \phi}\right)$. This equation demonstrates that $\varphi(r, \theta)$ is determined completely by its values $\varphi(a, \theta)$ on the restricted domain $r=a$. Hence we need only consider $r=a$, yielding the integral equation

$$
\mu \varphi(a, \theta)=a \int_{0}^{2 \pi} \mathcal{W}(a, a ; \phi) \varphi(a, \theta-\phi) d \phi .
$$

The solutions of this equation are exponential functions $e^{\gamma \theta}$, where $\gamma$ satisfies

$$
a \int_{0}^{2 \pi} \mathcal{W}(a, a ; \phi) e^{-\gamma \phi} d \phi=\mu
$$

By the requirement that $\varphi$ is $2 \pi$-periodic in $\theta$, it follows that $\gamma=i n$, where $n \in \mathbb{Z}$. Thus the integral operator with kernel $\mathcal{W}$ has a discrete spectrum given by

$$
\begin{aligned}
\mu_{n} & =a \int_{0}^{2 \pi} \mathcal{W}(a, a ; \phi) e^{-i n \pi \phi} d \phi \\
& =a \int_{0}^{2 \pi} w\left(\sqrt{a^{2}+a^{2}-2 a^{2} \cos \phi}\right) e^{-i n \phi} d \phi \\
& =2 a \int_{0}^{\pi} w(2 a \sin (\phi)) e^{-2 i n \phi} d \phi
\end{aligned}
$$

(after rescaling $\phi$ ). Note that $\mu_{n}$ is real since

$$
\operatorname{Im}\left\{\mu_{n}(a)\right\}=-2 a \int_{0}^{\pi} w(2 a \sin (\phi)) \sin (2 n \phi) d \phi=0 ;
$$


i.e., the integrand is odd-symmetric about $\pi / 2$. Hence,

$$
\mu_{n}(a)=\operatorname{Re}\left\{\mu_{n}(a)\right\}=2 a \int_{0}^{\pi} w(2 a \sin (\phi)) \cos (2 n \phi) d \phi
$$

with the integrand even-symmetric about $\frac{\pi}{2}$. Since $w(r)$ is a positive function of $r$, it follows that

$$
\mu_{n}(a) \leq 2 a \int_{0}^{\pi} w(2 a \sin (\phi))|\cos (2 n \phi)| d \phi \leq 2 a \int_{0}^{\pi} w(2 a \sin (\phi)) d \phi=\mu_{0}(a) .
$$

Given the set of solutions $\left\{\mu_{n}(a), n \geq 0\right\}$ for a pulse of width $a$ (assuming that it exists), we obtain from (5.18) the following pairs of eigenvalues:

$$
\lambda_{n}^{ \pm}=\frac{1}{2}\left(-\Lambda_{n} \pm \sqrt{\Lambda_{n}^{2}-4 \epsilon(1+\beta)\left(1-\Gamma_{n}\right)}\right),
$$

where

$$
\Lambda_{n}=1+\epsilon-\Gamma_{n}(1+\beta), \quad \Gamma_{n}=\frac{\mu_{n}(a)}{\left|U^{\prime}(a)\right|(1+\beta)} .
$$

Stability of the two-dimensional pulse requires that

$$
\Lambda_{n}>0, \quad \Gamma_{n}<1 \quad \text { for all } n \geq 0 .
$$

This reduces to the stability conditions

$$
\begin{array}{lll}
\epsilon>\beta: & \Gamma_{n}<1 & \text { for all } n \geq 0, \\
\epsilon<\beta: & \Gamma_{n}<\frac{1+\epsilon}{1+\beta} & \text { for all } n \geq 0 .
\end{array}
$$

Next we rewrite the stability conditions in terms of the gradient of the input $D(a)=\left|I^{\prime}(a)\right|$. From (5.2), (5.8), and (5.9) we have

$$
U^{\prime}(a)=\frac{1}{1+\beta}\left(-M_{r}(a)+I^{\prime}(a)\right)
$$

where

$$
M_{r}(a) \equiv-\left.\frac{\partial}{\partial r} M(a, r)\right|_{r=a}=2 \pi a \int_{0}^{\infty} \rho \breve{w}(\rho) J_{1}(a \rho) J_{1}(a \rho) d \rho .
$$

We have already established in section 5.1 that $M_{r}(a)>0$. Hence,

$$
\begin{aligned}
\left|U^{\prime}(a)\right| & =\left(\frac{1}{1+\beta}\right)\left|-M_{r}(a)+I^{\prime}(a)\right| \\
& =\left(\frac{1}{1+\beta}\right)\left(M_{r}(a)+D(a)\right) .
\end{aligned}
$$


The stability conditions (5.23) thus become

$$
\begin{array}{lll}
\epsilon>\beta: & D(a)>\mu_{n}(a)-M_{r}(a) & \text { for all } n \geq 0, \\
\epsilon<\beta: & D(a)>\left(\frac{1+\beta}{1+\epsilon}\right) \mu_{n}(a)-M_{r}(a) & \text { for all } n \geq 0 .
\end{array}
$$

Finally, using the fact that $\mu_{0}(a) \geq \mu_{n}(a)$ for all $n \geq 1$ and $M_{r}(a)>0$, we obtain the reduced stability conditions

$$
\begin{array}{ll}
\epsilon>\beta: & D(a)>\mu_{0}(a)-M_{r}(a) \equiv D_{\mathrm{SN}}(a), \\
\epsilon<\beta: & D(a)>\left(\frac{1+\beta}{1+\epsilon}\right) \mu_{0}(a)-M_{r}(a) \equiv D_{c}(a) .
\end{array}
$$

We now relate stability of the stationary pulse to the gradient $D$ on different branches of the existence curves shown in Figure 9 for $w(r)$ given by the exponential distribution (5.9). In this case the integral expressions for $\mu_{0}(a), M_{r}(a)$, and $\mathbf{M}(a)$ can be evaluated explicitly in terms of finite sums of modified Bessel and Struve functions; see Appendix B.

Stability for $\epsilon>\boldsymbol{\beta}$. Equation (5.10) implies that $G^{\prime}(a)=\mathbf{M}^{\prime}(a)+I^{\prime}(a)$. Since $\mathbf{M}(a)=$ $M(a, a)$, it follows from (5.3), (5.20), and (5.24) that

$$
\begin{aligned}
\mathbf{M}^{\prime}(a) & =\partial_{1} M(a, a)+\partial_{2} M(a, a) \\
& =\mu_{0}(a)-M_{r}(a),
\end{aligned}
$$

and hence

$$
G^{\prime}(a)=\mu_{0}(a)-M_{r}(a)-D(a) .
$$

We now see that the stability condition (5.26) is satisfied when $G^{\prime}(a)<0$ and is not satisfied when $G^{\prime}(a)>0$. Saddle-node bifurcations occur when $G^{\prime}(a)$, that is, when $D(a)$ passes through $D_{\mathrm{SN}}(a)=\mu_{0}(a)-M_{r}(a)$. This establishes the stability of the middle branch in case (i) and the upper branch of cases (ii) and (iii) shown in the left-hand column of Figure 9.

Hopf curves for $\epsilon<\boldsymbol{\beta}$. A Hopf bifurcation occurs when $\Lambda_{0}=0$ and $\Gamma_{0}<1$, or equivalently, when $D(a)=D_{c}(a)$. Since $\mu_{0}(a)>0$ it follows from (5.26) and (5.27) that $D_{c}(a)>D_{\mathrm{SN}}(a)$, and hence Hopf bifurcations occur only on the branches that are stable when $\epsilon>\beta$. As in the one-dimensional case, the Hopf and saddle-node points coincide when $\epsilon=\beta$, and so we expect, as $\epsilon$ decreases from $\beta$, the Hopf bifurcation point(s) to traverse these previously stable branches from the saddle-node point(s). Again, in order to show this more explicitly, we find a relationship for $D(a)$ that is independent of the input amplitude $\mathcal{I}$. Using (5.10), the input gradient $D$ can be related as

$$
\begin{aligned}
D(a) & =\left|I^{\prime}(a)\right| \\
& =\frac{a}{\sigma^{2}} I(a) \\
& =\frac{a}{\sigma^{2}}(\kappa(1+\beta)-\mathbf{M}(a)) .
\end{aligned}
$$

For each of the cases discussed in section 3.1, we examine graphically the crossings of the curves $D(a), D_{c}(a)$ : stability corresponds to $D(a)>D_{c}(a)$ with Hopf points at $D(a)=D_{c}(a)$. 

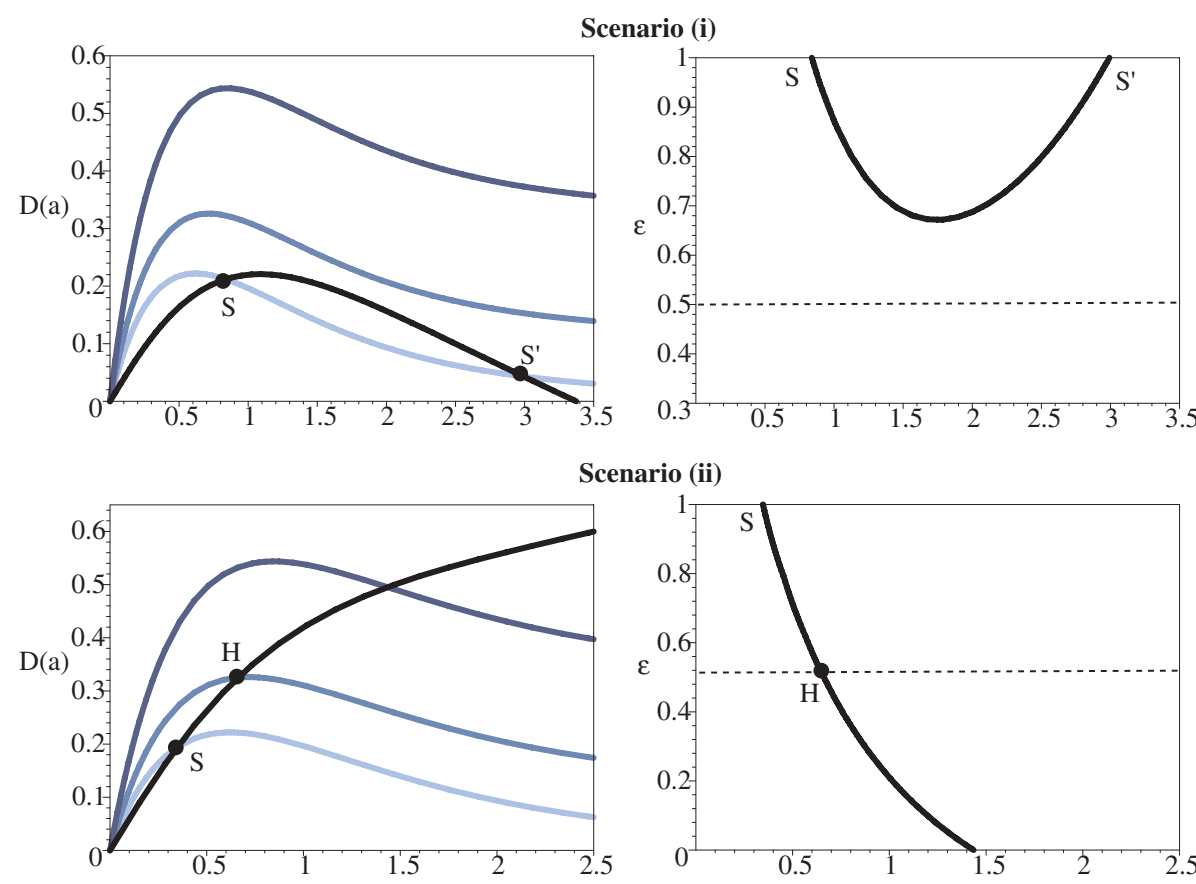

Scenario (ii)
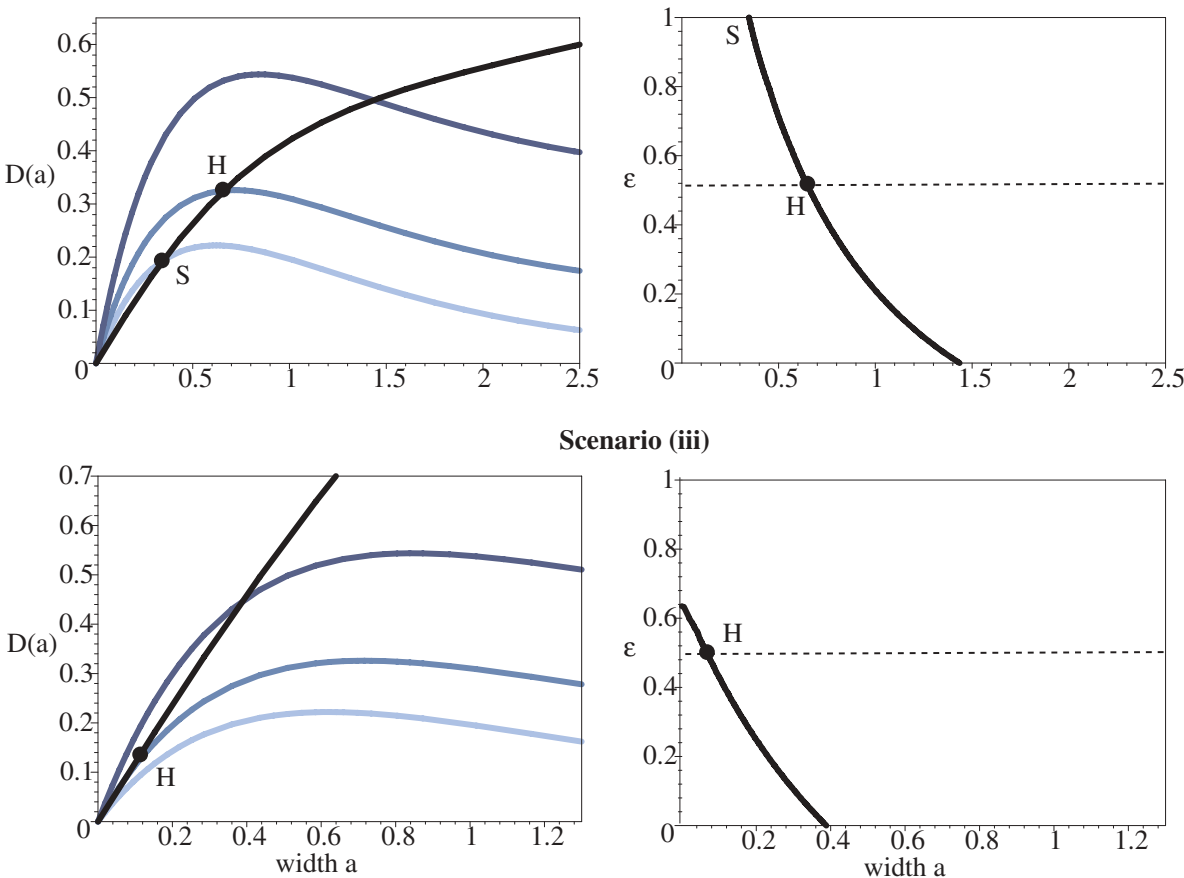

Scenario (iii)

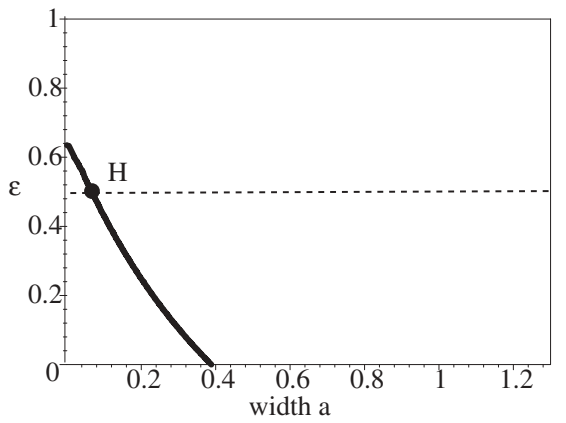

Figure 11. Left column: Gradient curves for the various bifurcation scenarios shown in Figure 9: (i) $\kappa_{c}<$ $\hat{\kappa}<\frac{1}{2}$, (ii) $\frac{1}{2}<\hat{\kappa}<\kappa_{0}$, and (iii) $\kappa_{0}<\hat{\kappa}$. The thick solid curve shows the input gradient $D(a)$ as a function of pulse width $a$. The lighter curves show the critical gradient $D_{c}(a)$ as function of a for $\epsilon=0.0,0.5,1.0$ and $\beta=1$. For a given value of $\epsilon<\beta$, a stationary pulse of width a is stable provided that $D(a)>D_{c}(a)$. A pulse loses stability via a Hopf bifurcation at the intersection points $D(a)=D_{c}(a)$. The Hopf bifurcation points for $\epsilon=0.5$ are indicated by $H$; in the first scenario there are no Hopf points at this particular value of $\epsilon$. In the limit $\epsilon \rightarrow \beta$, we have $H \rightarrow S$. Right column: Corresponding Hopf stability curves in the $(a, \epsilon)$-plane.

The results are displayed in Figure 11. Note that as in one dimension, sufficiently wide pulse solutions are always stable, as can be established by studying the asymptotic behavior of $D(a)$ and $D_{c}(a)$; see Appendix B.

Two-dimensional breathers. Analogous to the one-dimensional case, we find numerically that the upper branch in scenarios (ii) and (iii) can undergo a supercritical Hopf bifurcation leading to the formation of a two-dimensional breather. An example is shown in Figure 12, which was obtained using a Runge-Kutta scheme on a $300 \times 300$ grid with a time step of 0.02 . 

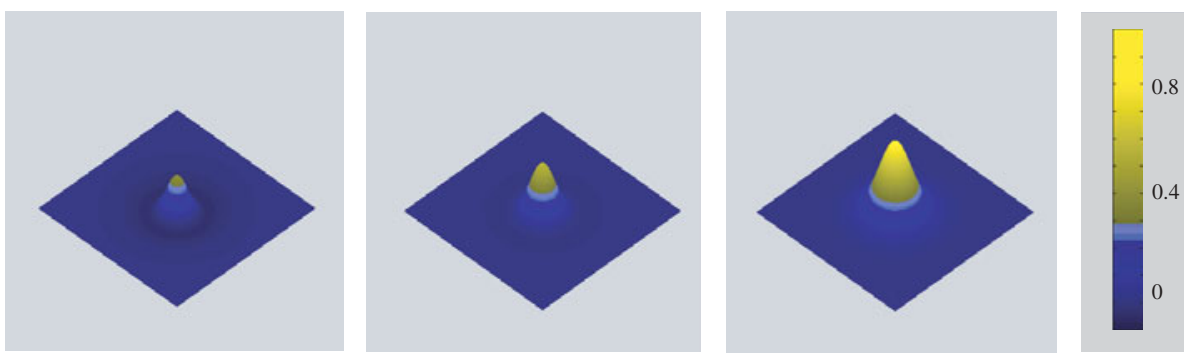

Figure 12. Two-dimensional breather with $\beta=4, \kappa=0.25, \epsilon=0.1, \mathcal{I}=0.26$. Clicking on the above image displays the associated movie.
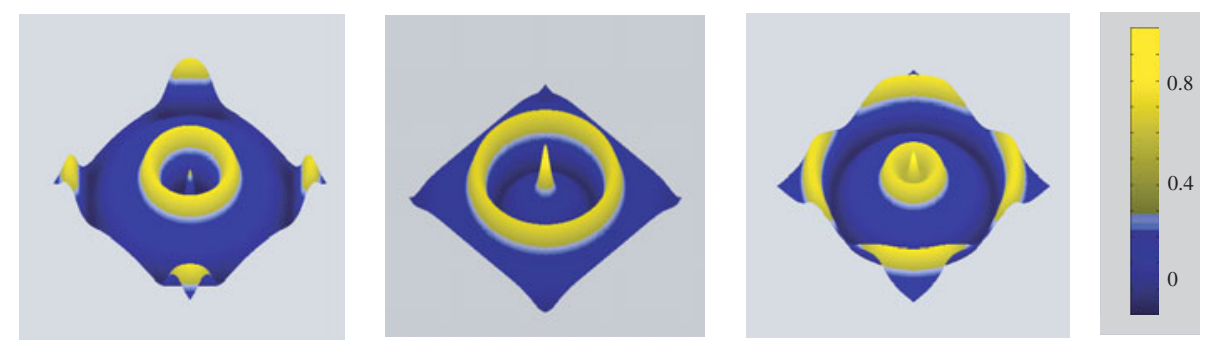

Figure 13. Two-dimensional pulse emitter with $\beta=4, \kappa=0.2, \epsilon=0.1, \mathcal{I}=0.2$. Clicking on the above image displays the associated movie.

Moreover, the breather can undergo a secondary instability, resulting in the periodic emission of circular target waves; see Figure 13.

6. Discussion. In this paper we have shown that a localized external input can induce oscillatory behavior in an excitatory neural network in the form of breathing pulses and that these breathers can subsequently act as sources of wave emission. Interestingly, following some initial excitation, breathers can be supported by subthreshold inputs. From a mathematical perspective, there are a number of directions for future work. First, one could try to develop some form of weakly nonlinear analysis in order to determine analytically whether or not the Hopf instability of the stationary pulse is supercritical or subcritical. It would also be interesting to explore more fully the behavior around the degenerate bifurcation point $\epsilon=\beta$, where there exists a pair of zero eigenvalues of the associated linear operator that is suggestive of a Takens-Bogdanov bifurcation. The latter would predict that for certain parameter values around the degenerate bifurcation point, the periodic orbit arising from the Hopf bifurcation could be annihilated in a homoclinic bifurcation associated with another unstable stationary pulse. This could provide one mechanism for the disappearance of the breather as the amplitude of the external input is reduced. (A pulse-emitter does not occur when $\epsilon \approx \beta$.) Another extension is to consider a smooth nonlinear output function $f(u)=1 /\left(1+\mathrm{e}^{-\gamma(u-\kappa)}\right)$, which reduces to the Heaviside function in the high gain limit $\gamma \rightarrow \infty$. In the case of sufficiently slow adaptation (small $\epsilon$ ), it might be possible to use singular perturbation methods along the lines of Pinto and Ermentrout [25], who established the existence of traveling pulses in a homogeneous network with smooth $f$. Finally, it would be interesting to extend our analysis to the case of traveling waves locking to a moving stimulus; the associated stability analysis 
would then involve Evans functions. From an experimental perspective, our results could be tested by introducing an inhomogeneous current into a cortical slice and searching for these oscillations. One potential difficulty of such an experiment is that persistent currents tend to burn out neurons. An alternative approach might be to use some form of pharmacological manipulation of NMDA receptors, for example, or an application of an external electric field that modifies the effective threshold of the neurons. Note that the usual method for inducing traveling waves in cortical slices (and in corresponding computational models) is to introduce short-lived current injections; once the wave is formed it propagates in a homogeneous medium.

Appendix A. We used the following biophysical model functions and parameter values in section 4.2:

$$
\begin{aligned}
& V_{\mathrm{syn}}=-45 \mathrm{mV} \text {, } \\
& g_{\text {syn }}=20 \mathrm{mS} / \mathrm{cm}^{2} \text {, } \\
& V_{\mathrm{K}}=-100 \mathrm{mV} \text {, } \\
& g_{\mathrm{K}}=80 \mathrm{mS} / \mathrm{cm}^{2} \text {, } \\
& V_{\mathrm{Na}}=50 \mathrm{mV} \text {, } \\
& g_{\mathrm{Na}}=100 \mathrm{mS} / \mathrm{cm}^{2} \text {, } \\
& V_{\mathrm{L}}=-67 \mathrm{mV} \text {, } \\
& g_{\mathrm{L}}=0.2 \mathrm{mS} / \mathrm{cm}^{2} \text {, } \\
& F=1 \mu \mathrm{F} / \mathrm{cm}^{2} \text {, } \\
& g_{q}=3 \mathrm{mS} / \mathrm{cm}^{2} \text {, } \\
& \alpha_{m}(v)=0.32(54+v) /(1-\exp (-(v+54) / 4)), \\
& \beta_{m}(v)=0.28(v+27) /(\exp ((v+27) / 5)-1), \\
& \alpha_{h}(v)=0.128 \exp (-(50+v) / 18) \text {, } \\
& \beta_{h}(v)=4 /(1+\exp (-(v+27) / 5)), \\
& \alpha_{n}(v)=0.032(v+52) /(1-\exp (-(v+52) / 5)) \text {, } \\
& \beta_{n}(v)=0.5 \exp (-(57+v) / 40) \text {, }
\end{aligned}
$$

where

$$
\begin{gathered}
p_{\infty}(v)=\frac{\alpha_{p}(v)}{\alpha_{p}(v)+\beta_{p}(v)}, \quad \tau_{p}(v)=\frac{1}{\alpha_{p}(v)+\beta_{p}(v)}, \quad p \in\{m, n, h\} \\
q_{\infty}(v)=\frac{1}{1+e^{(-(v+35) / 20)}}, \quad \tau_{q}(v)=\frac{1000}{3.3 e^{(v+35) / 20}+e^{-(v+35) / 20}} \\
\tau=1, \quad K(V)=\frac{1}{1+e^{-(V+50)}}
\end{gathered}
$$

Appendix B. In this appendix we evaluate the gradient functions $D(a)$ and $D_{c}(a)$ of $(5.30)$ and (5.27) for the exponential weight distribution (5.9). We then determine their asymptotic behavior for large pulse width $a$, thus establishing the stability of stationary pulses in the 
limit $a \rightarrow \infty$. First, from (5.20), we can write

$$
\begin{aligned}
\mu_{0}(a) & =\frac{a}{\pi} \int_{0}^{\pi} \exp (-2 a \sin \phi) d \phi \\
& =\frac{2 a}{\pi} \int_{0}^{\frac{\pi}{2}} \exp (-2 a \cos \phi) d \phi \\
& =a\left(\frac{2}{\pi} \int_{0}^{\frac{\pi}{2}} \cosh (-2 a \cos \phi) d \phi\right)-a\left(\frac{2}{\pi} \int_{0}^{\frac{\pi}{2}} \sinh (-2 a \cos \phi) d \phi\right) \\
& =a\left(I_{0}(2 a)-\mathbf{L}_{0}(2 a)\right)
\end{aligned}
$$

where $I_{\nu}$ is a modified Bessel function and $\mathbf{L}_{\nu}$ denotes a modified Struve function [33]. Second, from (5.24),

$$
\begin{aligned}
M_{r}(a) & =a \int_{0}^{\infty} \frac{\rho}{\left(\rho^{2}+1\right)^{\frac{3}{2}}} J_{1}(a \rho) J_{1}(a \rho) \\
& =a\left(\mathbf{L}_{0}(2 a)-I_{0}(2 a)\right)-\left(\mathbf{L}_{1}(2 a)-I_{1}(2 a)\right),
\end{aligned}
$$

where

$$
I_{1}(2 a)=\frac{4 a}{\pi} \int_{0}^{\pi / 2} \cosh (2 a \cos \theta) \sin ^{2} \theta d \theta
$$

and

$$
\mathbf{L}_{1}(2 a)=\frac{4 a}{\pi} \int_{0}^{\pi / 2} \sinh (2 a \cos \theta) \sin ^{2} \theta d \theta
$$

Equation (5.27) then implies that

$$
D_{c}(a)=\frac{\beta+\epsilon+2}{1+\epsilon} a\left(I_{0}(2 a)-\mathbf{L}_{0}(2 a)\right)-\left(I_{1}(2 a)-\mathbf{L}_{1}(2 a)\right) .
$$

Using the asymptotic expansions for large $a$,

$$
\begin{aligned}
& I_{0}(2 a)-\mathbf{L}_{0}(2 a) \sim \frac{1}{\pi}\left(\frac{1}{a}+\frac{1}{4 a^{2}}\right), \\
& I_{1}(2 a)-\mathbf{L}_{1}(2 a) \sim \frac{2}{\pi}\left(1-\frac{1}{4 a^{2}}\right),
\end{aligned}
$$

we deduce that

$$
D_{c}(a) \sim \frac{1}{\pi}\left(\frac{\beta-\epsilon}{1+\epsilon}\right)+\left(\frac{1}{4}\left(\frac{\beta-\epsilon}{1+\epsilon}\right)+1\right) \frac{1}{\pi a^{2}} .
$$

Similarly, from (5.11) we have

$$
\begin{aligned}
\mathbf{M}(a) & =a \int_{0}^{\infty} \frac{1}{\left(\rho^{2}+1\right)^{\frac{3}{2}}} J_{0}(a \rho) J_{1}(a \rho) d \rho \\
& =\left(\frac{1}{2}+a I_{1}(2 a)-\frac{1}{2} I_{0}(2 a)\right)-\left(\frac{2 a}{\pi}+a \mathbf{L}_{1}(2 a)-\frac{1}{2} \mathbf{L}_{0}(2 a)\right) .
\end{aligned}
$$


Equation (5.30) and the asymptotic expansions (B.5) then imply that

$$
D(a) \sim \frac{a}{\sigma^{2}}\left((1+\beta) \kappa-\frac{1}{2}\right)+\frac{1}{\pi \sigma^{2}}-\frac{1}{8 \pi a^{2}} .
$$

Finally, combining (B.6) and (B.8),

$$
D(a)-D_{c}(a) \sim \frac{a}{\sigma^{2}}\left((1+\beta) \kappa-\frac{1}{2}\right)+\frac{1}{\pi \sigma^{2}}-\left(\frac{\beta-\epsilon}{1+\epsilon}\right)+\mathcal{O}\left(a^{-2}\right) .
$$

From this we conclude that for all $\sigma>0$, a stationary-pulse solution (if it exists) is stable in the limit $a \rightarrow \infty$, provided that $\hat{\kappa}>1 / 2$.

\section{REFERENCES}

[1] S. Amari, Dynamics of pattern formation in lateral inhibition type neural fields, Biol. Cybernetics, 27 (1977), pp. $77-87$.

[2] M. BoDE, Front-bifurcations in reaction-diffusion systems with inhomogeneous parameter distributions, Phys. D, 106 (1997), pp. 270-286.

[3] W. H. Bosking, Y. Zhang, B. Schofield, and D. Fitzpatrick, Orientation selectivity and the arrangement of horizontal connections in tree shrew striate cortex, J. Neurosci., 17 (1997), pp. 21122127.

[4] P. C. BRessloff, Traveling fronts and wave propagation failure in an inhomogeneous neural network, Phys. D, 155 (2001), pp. 83-100.

[5] P. C. Bressloff, S. E. Folias, A. Pratt, and Y.-X. Li, Oscillatory waves in inhomogeneous neural media, Phys. Rev. Lett., 91 (2003), 178101.

[6] P. C. Bressloff And S. E. Folias, Front bifurcations in an excitatory neural network, SIAM J. Appl. Math., 65 (2004), pp. 131-151.

[7] R. D. Chervin, P. A. Pierce, And B. W. Connors, Propagation of excitation in neural network models, J. Neurophysiol., 60 (1988), pp. 1695-1713.

[8] G. B. ERmentrout And J. B. MCLeOd, Existence and uniqueness of travelling waves for a neural network, Proc. Roy. Soc. Edinburgh Sect. A, 123 (1993), pp. 461-478.

[9] G. B. Ermentrout, The analysis of synaptically generated traveling waves, J. Comput. Neurosci., 5 (1998), pp. 191-208.

[10] G. B. Ermentrout, Neural networks as spatial pattern forming systems, Rep. Progr. Phys., 61 (1998), pp. 353-430.

[11] G. B. Ermentrout And D. Kleinfeld, Traveling electrical waves in cortex: Insights from phase dynamics and speculation on a computational role, Neuron, 29 (2001), pp. 33-44.

[12] D. Golomb And Y. Amitai, Propagating neuronal discharges in neocortical slices: Computational and experimental study, J. Neurophysiol., 78 (1997), pp. 1199-1211.

[13] A. Hagberg And E. Meron, Pattern formation in nongradient reaction-diffusion systems: The effects of front bifurcations, Nonlinearity, 7 (1994), pp. 805-835.

[14] A. Hagberg, E. Meron, I. Rubinstein, and B. Zaltzman, Controlling domain patterns far from equilibrium, Phys. Rev. Lett., 76 (1996), pp. 427-430.

[15] M. A. P. Idiart And L. F. Аввотt, Propagation of excitation in neural network models, Network, 4 (1993), pp. 285-294.

[16] J. P. KeEner, Propagation of waves in an excitable medium with discrete release sites, SIAM J. Appl. Math., 61 (2000), pp. 317-334.

[17] J. P. KeEneR, Homogenization and propagation in the bistable equation, Phys. D, 136 (2000), pp. 1-17.

[18] D. Kleinfeld, K. R. Delaney, M. S. Fee, J. A. Flores, D. W. Tank, and A. Galperin, Dynamics of propagating waves in the olfactory network of a terrestrial mollusk: An electrical and optical study, J. Neurophysiol., 72 (1994), pp. 1402-1419. 
[19] C. R. Laing, W. C. Troy, B. Gutkin, and G. B. Ermentrout, Multiple bumps in a neuronal model of working memory, SIAM J. Appl. Math., 63 (2002), pp. 62-97.

[20] C. R. Laing And W. C. Troy, PDE methods for nonlocal models, SIAM J. Appl. Dyn. Syst., 2 (2003), pp. $487-516$.

[21] Y. W. Lam, L. B. Cohen, M. Wachowiak, and M. R. Zochowski, Odors elicit three different oscillations in the turtle olfactory bulb, J. Neurosci., 20 (2000), pp. 749-762.

[22] Y.-X. LI, Tango waves in a bidomain model of fertilization calcium waves, Phys. D, 186 (2003), pp. 27-49.

[23] R. Malach, Y. Amir, M. Harel, And A. Grinvald, Relationship between intrinsic connections and functional architecture revealed by optical imaging and in vivo targeted biocytin injections in primate striate cortex, Proc. Natl. Acad. Sci. USA, 90 (1993), pp. 10469-10473.

[24] M. A. L. Nicolelis, L. A. Baccala, R. C. S. Lin, And J. K. Chapin, Sensorimotor encoding by synchronous neural ensemble activity at multiple levels of the somatosensory system, Science, 268 (1995), pp. 1353-1358.

[25] D. J. Pinto And G. B. Ermentrout, Spatially structured activity in synaptically coupled neuronal networks: I. Traveling fronts and pulses, SIAM J. Appl. Math., 62 (2001), pp. 206-225.

[26] A. Prat And Y.-X. Li, Stability of front solutions in inhomogeneous media, Phys. D, 186 (2003), pp. 50-68.

[27] J. C. Prechtl, L. B. Cohen, B. Pasaram, P. P. Mitra, and D. Kleinfeld, Visual stimuli induce waves of electrical activity in turtle cortex, Proc. Natl. Acad. Sci. USA, 94 (1997), pp. 7621-7626.

[28] J. Rinzel And D. TeRman, Propagation phenomena in a bistable reaction-diffusion system, SIAM J. Appl. Math., 42 (1982), pp. 1111-1137.

[29] P. R. Roelfsema, A. K. Engel, P. Konig, And W. Singer, Visuomotor integration is associated with zero time-lag synchronization among cortical areas, Nature, 385 (1997), pp. 157-161.

[30] J. E. Rubin AND W. C. TROY, Sustained spatial patterns of activity in neuronal populations without recurrent excitation, SIAM J. Appl. Math., 64 (2004), pp. 1609-1635.

[31] P. Schutz, M. Bode, And H.-G. Purwins, Bifurcations of front dynamics in a reaction-diffusion system with spatial inhomogeneities, Phys. D, 82 (1995), pp. 382-397.

[32] J. G. TAYLOR, Neural "bubble" dynamics in two dimensions: Foundations, Biol. Cybernetics, 80 (1999), pp. 303-409.

[33] G. N. Watson, A Treatise on the Theory of Bessel Functions, Cambridge University Press, Cambridge, UK, 1952.

[34] H. Werner And T. Richter, Circular stationary solutions in two-dimensional neural fields, Biol. Cybernetics, 85 (2001), pp. 211-217.

[35] J.-Y. WU, L. GUAN, AND Y. TSAU, Propagating activation during oscillations and evoked responses in neocortical slices, J. Neurosci., 19 (1999), pp. 5005-5015.

[36] H. R. Wilson And J. D. Cowan, A mathematical theory of the functional dynamics of cortical and thalamic nervous tissue, Kybernetik, 13 (1973), pp. 55-80.

[37] T. Yoshioka, G. G. Blasdel, J. B. LevitT, And J. S. Lund, Relation between patterns of intrinsic lateral connectivity, ocular dominance, and cytochrome oxidase-reactive regions in macaque monkey striate cortex, Cerebral Cortex, 6 (1996), pp. 297-310. 